\title{
TRIM2 regulates the development and metastasis of tumorous cells of osteosarcoma
}

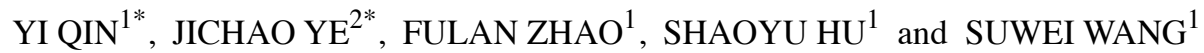 \\ ${ }^{1}$ Department of Orthopedics, Zhuhai Hospital, Jinan University, Zhuhai People's Hospital, Zhuhai, Guangdong 519000; \\ ${ }^{2}$ Department of Orthopedics, Sun Yat-Sen Memorial Hospital, Sun Yat-Sen University, \\ Guangzhou, Guangdong 510120, P.R. China
}

Received January 12, 2018; Accepted June 14, 2018

DOI: $10.3892 /$ ijo.2018.4494

\begin{abstract}
The present study aimed to investigate candidate genes involved in the development and metastasis of osteosarcoma. Candidate genes were screened preliminarily from the Gene Expression Omnibus database and then validated using actual tumor tissues collected from patients with osteosarcoma. The cells were prepared and transfected with specific gene-targeted small interfering RNA followed by an MTS assay for cell viability detection and Transwell assays for cell migration and invasion capacity detection. The cell apoptosis was determined by flow cytometry and the protein level of the genes was detected by western blot analysis. An in vivo nude model was used and injected with cells to detect the functions of the genes. Transcriptome sequencing was performed to verify the regulation network, followed by reverse transcription-quantitative polymerase chain reaction and western blot analyses for validation. Increased tripartite motif-containing protein 2 (TRIM2) was detected in the osteosarcoma tumor tissues compared with normal tissues. The inhibition of TRIM2 induced lower cell viability and cell invasion capacity, and increased the rate of cell apoptosis. Decreased TRIM2 also inhibited the development and metastasis of osteosarcoma in the nude mouse models. The transcriptome sequencing revealed that the regulation of TRIM2 may be correlated with genes, Sirtuin 4, DNA damage inducible transcript 3, cAMP responsive element binding protein $5, \mathrm{G}$ proteincoupled receptor 65 (GPR65) and ADP-ribosyltransferase 5. Western blot analysis indicated that TRIM2 regulated the development and metastasis of osteosarcoma via the phos-
\end{abstract}

Correspondence to: Dr Yi Qin, Department of Orthopedics, Zhuhai Hospital, Jinan University, Zhuhai People's Hospital, 79 Kangning Road, Zhuhai, Guangdong 519000, P.R. China

E-mail:kcqa41@163.com

*Contributed equally

Key words: osteosarcoma, metastasis, TRIM2, expression profile, regulation phoinositide 3-kinase/protein kinase B signaling pathway. Therefore, TRIM2 performs important functions in regulating the development and metastasis of osteosarcoma.

\section{Introduction}

Osteosarcoma is a primary mesenchymal tumor, which is considered an aggressive type of malignancy. Metastasis is an important process in malignant tumor formation $(1,2)$. Conventional chemotherapy is not efficient in patients with osteosarcoma, and $\sim 80 \%$ of patients with distant metastasis develop lung metastasis $(3,4)$. Evidence has shown that $10-20 \%$ of patients with osteosarcoma are at risk of developing pulmonary metastasis, and $\sim 40-50 \%$ of the patients develop pulmonary heterochrony although without metastasis (5). By contrast, patients with osteosarcoma with metastasis usually present with a lower 5-year survival rate, compared with those patients without metastasis (6). Conventional chemotherapy is not efficient for treatment of the disease, and $30-50 \%$ of patients eventually succumb to mortality as a result of the pulmonary metastasis (7). As the occurrence and development of the disease is closely associated with molecular biology, investigating the molecular mechanism of osteosarcoma has significant value for developments in early diagnosis and treatment.

The underlying biological mechanism of osteosarcoma tumors and metastasis has been widely investigated, and genes and pathways involved in osteosarcoma have been identified. For example, Notch proteins are important for normal bone development and homeostasis, and also for angiogenesis $(8,9)$. Dysregulation of the Notch pathway is present in human diseases associated with congenital skeletal abnormalities. The Notch pathway genes have been found to be overexposed in osteosarcoma cells, compared with normal bone cells, which include Hes related family bHLH transcription factor with YRPW motif 1 (HEY1), Hes family BHLH transcription factor 1 (HES1) and NOTCH2 (10-12). Runt related transcription factor 2 (RUNX2) is an important transcription factor involved in osteoblast differentiation (13). Previous studies have suggested that the Notch pathway transcription of Runx2 is decreased via HES and HEY proteins. As a result, it is hypothesized that the increase of immature osteoblasts may be one of the potential factors in malignant transformation $(14,15)$. 
The Wnt pathway manages several processes, including cell growth, normal bone development and carcinogenesis $(16,17)$. According to previous studies, the Wnt pathway was found to be highly activated in human osteosarcoma, therefore, it may be also potential pathway involved in osteosarcoma (18-20). Mammalian target of rapamycin (mTOR), one of the phosphoinositide 3-kinase (PI3K) family members, is important in the regulation of cell cycle, growth and development $(21,22)$. It was shown that, by suppressing the expression of mTOR or phosphatidylinositol-4,5-bisphosphate 3-kinase catalytic subunit $\alpha$, growth arrest was induced in a murine osteosarcoma model (23). Based on the evidence so far, molecular regulation appears to be important in the occurrence and development of osteosarcoma. The investigation of genes which are correlated with osteosarcoma are likely to be beneficial for the diagnosis and treatment of the disease.

RNA sequencing (RNA-seq) is an advanced technique based on the deep-sequencing approach. It provides a relatively unbiased and more precise measurement for the quantitation of the transcripts and their isoforms (24). As RNA-seq technology presents apparent advantages against conventional hybridization-based microarrays, it has been widely applied and utilized in the screening the differentially expressed genes in multiple experimental conditions. In the present study, candidate genes involved in osteosarcoma were screened by analyzing the Gene Expression Omnibus (GEO) dataset. A large number of differentially expressed genes were screened based on the results of the microarray (https:/www.ncbi.nlm. nih.gov/geo/query/acc.cgi?acc=GSE21257). The present study also verified the differentially expressed genes, which have not been reported in osteosarcoma, in six cell lines (hFOB, SW1353, SAOS-2, MG-63, 143B and U2-OS) by reverse transcription-quantitative polymerase chain reaction (RT-qPCR) analysis. The results indicated that the expression levels of Kelch domain containing 1 (KLHDC1), TRIM2, Laccase domain-containing protein 1 (LACC1), Mitochondrial transcription factor A (TFAM), Laminin subunit $\beta 1$ (LAMB1), Stanniocalcin 2 (STC2) and Vimentin (VIM) in the osteosarcoma cell lines were significantly higher than those in normal osteoblasts (data not shown). As the TRIM2, STC2 and VIM genes were significantly expressed in osteosarcoma cells, they were selected for metastatic analysis and analysis of prognosis. The prognosis and metastasis analyses indicated that TRIM2 was important in the pathogenic process. Relevant cell assays and RNA-seq were performed in order to verify the mechanism underlying the effect of TRIM2.

\section{Materials and methods}

Prognosis and metastasis analysis. The expression profiles and clinical data of the patients with osteosarcoma were downloaded from the NCBI GEO database (accession no. GSE21257). The methods of pretreatment of specimens, RNA isolation, and microarray assay setup were described on the website. The expression values were used for statistical analysis. The expression differences are presented via box-plots. Differences in overall survival rates between 'high' and 'low' expression groups were compared using Kaplan-Meier curves, with P-values calculated via the log-rank test, using the Survival package in R version 3.2.3 (https://www.r-project.org/).
Table I. Clinicopathological information of the 53 patients with osteosarcoma.

Characteristic

$\mathrm{n}$

$\begin{array}{lr}\text { Age (years) } & \\ <20 & 42 \\ >20 & 11 \\ \text { Sex } & \\ \text { Male } & 34 \\ \text { Female } & 19 \\ \text { Distant metastasis } & \\ \text { Absent } & 19 \\ \text { Present } & 34 \\ \text { Huvos grade } & \\ \text { Unknown } & 6 \\ 1 & 13 \\ 2 & 16 \\ 3 & 13 \\ 4 & 5\end{array}$

Status

Alive $\quad 30$

Deceased $\quad 23$

Immunohistochemical staining. Fresh primary osteosarcoma samples and paired adjacent non-tumor soft tissues from 53 patients undergoing surgical resection at Zhuhai Hospital, Jinan University (Zhuhai, China) between April 2010 and June 2011 were collected, formalin-fixed and paraffin-embedded. The present study was approved by the Institutional Research Ethics Committee of Jinan University. Informed consent was provided by all participants prior to the study. The clinicopathological information of the 53 patients were recorded and are presented in Table I.

The formalin-fixed and paraffin-embedded (FFPE) tissue sections $(5 \mu \mathrm{m})$ were subjected to antibody thermal remediation, the activity of endogenous peroxidase was inhibited by hydrogen peroxide and then closed with goat or mouse serum (Gibco; Thermo Fisher Scientific, Inc., Waltham, MA, USA) following dewaxing and hydration. The primary antibodies TRIM2 (cat. no. ab3942; 1:1,000), E-cadherin (cat. no. ab76055; 1:1,000), N-cadherin (cat. no. ab18203; 1:500) or Vimentin (cat. no. ab92547, 1:500) (all from Abcam, Cambridge, UK) diluted in 1X PBS solution were used to cover the tissue sections and incubated overnight at $4^{\circ} \mathrm{C}$. Anti-mouse immunoglobulin $\mathrm{G}(\mathrm{IgG})$ (cat. no. sc-516102; 1:2,000), anti-rabbit IgG (cat. no. sc-3753; 1:500) or anti-goat IgG (cat. no. sc-2489; 1:500) (all from Santa Cruz Biotechnology, Inc., Dallas, TX, USA) secondary antibodies were used to bind the specific primary antibodies at $37^{\circ} \mathrm{C}$ for $30 \mathrm{~min}$, and DAB was applied for coloration. The stained tissues were observed using IX71 inverted microscopy (Olympus Corporation, Tokyo, Japan). Six samples for each group were used for the immunohistochemical staining.

Cell culture. The hFOB, U2-OS, SW1353, MG63, 143B and SAOS-2 human osteosarcoma cell lines provided by American 
Type Culture Collection (Manassas, VA, USA) were used in the present study. The cells were incubated in DMEM (Gibco; Thermo Fisher Scientific, Inc.). The medium was supplemented with $10 \%$ FBS (Gibco; Thermo Fisher Scientific, Inc.) and the cells were all maintained at $37^{\circ} \mathrm{C}$ in $5 \% \mathrm{CO}_{2}$. On reaching $70-80 \%$ confluence, the cells were washed with PBS and detached with $0.25 \%$ trypsin/0.2\% EDTA. Cell morphology was viewed under a light microscope, and suspended to the concentration of $1 \times 10^{6} / \mathrm{ml}$.

Lentivirus production and transfection. The night prior to transfection, 3x10 $293 \mathrm{~T}$ (CRL-3216 ${ }^{\mathrm{TM}}$; American Type Culture Collection, Manassas, VA, USA) cells were seeded into $10 \mathrm{~cm}$ dishes. Small interfering RNA (siRNA) sequences targeted to TRIM2 were cloned into the PLKO.1 lentiviral vector (Dharmacon, Inc., Lafayette, CO, USA). The cells were transfected with the TRIM2 siRNA-expressing PLKO.1 lentiviral vectors and control empty vector along with packaging vectors (psPAX2 and pMD2.G) using FuGENE-6 transfection reagent (Promega Corporation, Madison, WI, USA). The lentiviruscontaining supernatants were harvested $72 \mathrm{~h}$ following transfection and were filtered through $0.45-\mu \mathrm{m}$ PVDF filters. The supernatant was then concentrated by ultracentrifugation (at $100,000 \mathrm{x} g$ for $2 \mathrm{~h}$ at room temperature) in a Beckman Optima L-90K ultracentrifuge (Beckman Coulter, Inc., Brea, CA, USA). The virus-containing pellet was dissolved in DMEM, aliquoted and stored at $-80^{\circ} \mathrm{C}$. The sequences of the siRNAs were as follows: siTRIM2-1 (human), forward, 5'-CCTGGAACG GTACAAGAAT-3' and reverse, 5'-ATTCTTGTACCGTTCC AGG-3'; siTRIM2-2 (human), forward, 5'-CCAGTGAAGGCA CCAACAT-3' and reverse, 5'-ATGTTGGTGCCTTCACTGG-3'.

The U2OS and MG63 cells were infected by adding $1 \mathrm{ml}$ of concentrated virus supplemented with $2 \mu \mathrm{g} / \mathrm{ml}$ polybrene to $4 \times 10^{5}$ cells in 12 -well plates. After $36 \mathrm{~h}$ at room temperature, the viral supernatant was replaced with standard growth medium, and transduction efficiency was monitored by GFP expression $48 \mathrm{~h}$ following replacement of the virus-containing medium with normal medium.

Cell viability assay. Cell viability was examined using the MTS included in the CellTiter 96 Aqueous One Solution Cell Proliferation Assay (Promega Corporation) according to the manufacturer's protocol. The U2OS and MG63 cells were seeded in a 96-well plate at a cell density of 200 cells per well. The cells were incubated with $15 \mu \mathrm{l}$ of MTS reagent solution for $4 \mathrm{~h}$ and the produced formazan was measured at $490 \mathrm{~nm}$ in a Sunrise Basic Tecan cell plate reader with Magellan 6 software (both from Tecan Group, Ltd., Grödig, Austria).

Transwell migration and invasion assays. For the Transwell migration assays, $2.5-5 \times 10^{4}$ cells were plated in the top chamber with a non-coated membrane $(8-\mu \mathrm{m}$ pore size; Corning Incorporated, Corning, NY, USA). For the cell invasion assay, the Transwell chambers were pretreated with Matrigel (EMD Millipore, Billerica, MA, USA) and the cells were resuspended at a density of $2 \times 10^{5}$ cells $/ \mathrm{ml}$ in serum-free medium at $48 \mathrm{~h}$ post-transfection. In the two assays, the upper chamber was filled with $300 \mu 1$ cell suspensions and the lower well was filled with complete medium. After $24 \mathrm{~h}$, the cells in the upper chambers were removed with a cotton swab, and the migrated or invasive cells in the lower chambers were fixed with $4 \%$ formaldehyde and stained with crystal violet. The cells were observed and counted by IX71 inverted microscopy (Olympus Corporation).

Cell apoptosis assay. The cells were seeded in 6-well plates and incubated for $48 \mathrm{~h}$. The cells were detached with trypsin and the apoptosis assay was performed using an Annexin V assay kit according to the manufacturer's protocol. The assay was performed with a BD FACSCanto II flow cytometer (BD Biosciences, Franklin Lakes, NJ, USA). The apoptosis data were analyzed with FACSCanto II flow cytometer software (BD Biosciences).

RNA-seq. Total RNA was extracted with TRIzol reagent (Invitrogen; Thermo Fisher Scientific, Inc.) according to the manufacturer's protocol. mRNA was isolated from the total RNA using oligo(dT) magnetic beads, and fragmentation buffer was subsequently added for the fragmentation of mRNA. The mRNA fragments were subsequently used as templates for first-strand cDNA synthesis using random primers. The second-strand cDNA was synthesized using DNA polymerase I. Adaptors were ligated to the fragments and 200-bp fragments were subsequently enriched by PCR amplification. The quality of the library was confirmed using the Agilent 2100 Bioanalyzer. The sequencing was performed on the Hiseq2000 platform.

The primary raw reads were trimmed of adaptors and filtered to remove low quality reads using SOAP nuke software version 1.5 .3 (http://soap.genomics.org.cn). The length of raw reads used were 150-bp in pair end. The cleaned reads were aligned to the hg19 reference genome using hierarchical indexing for spliced alignment of transcripts. DEGseq2 were used to detect the differentially expressed genes. The P-value threshold was determined by the false discovery rate (FDR) using an FDR threshold $\leq 0.001$. Gene Ontology (GO) functional enrichment and Kyoto Encyclopedia of Genes and Genomes (KEGG) pathway analysis were performed using the Blast2GO program version 5.1 (https://www.blast2go. $\mathrm{com} /$ ).

$R T$-qPCR assay. Total RNA was prepared using TRIzol (Invitrogen; Thermo Fisher Scientific, Inc.) following the manufacturer's protocol. The genomic DNA-free RNA was then converted into cDNA using M-MLV Reverse Transcriptase (Promega Corporation), according to the manufacturer's protocol. PCR was carried out on the CFX96 system (Bio-Rad Laboratories, Inc., Hercules, CA, USA) in a $20 \mu 1$ reaction mixture containing $10 \mu \mathrm{l} \mathrm{GoTaq}{ }^{\circledR}$ Green Master Mix (Promega Corporation), $1.5 \mu \mathrm{l}$ cDNA, $0.5 \mu \mathrm{l}$ each primer and $7.5 \mu \mathrm{l}$ nuclease-free water under the following conditions: Initial denaturation at $95^{\circ} \mathrm{C}$ for $120 \mathrm{sec}$, followed by 40 cycles of denaturation at $95{ }^{\circ} \mathrm{C}$ for $15 \mathrm{sec}$ and extension at $60^{\circ} \mathrm{C}$ for $30 \mathrm{sec}$. The primer sequences were as follows: Sirtuin 4 (SIRT4), forward, $5^{\prime}-\mathrm{AAG}$ CCTCCATTGGGTTATTTGT-3' and reverse, 5'-TGTAGTCTG GTATCCCCGATTC-3'; DNA damage-inducible transcript 3 (DDIT3), forward, 5'-GAACGGCTCAAGCAGGAA ATC-3' and reverse, 5'-ATTCACCATTCGGTCAATCAGAG-3'; cAMP responsive element binding protein 5 (CREB5), forward, 
5'-AAAGACTGCCCAATAACAGCC-3' and reverse, 5'-AAG CTGGGACAGGACTAGCA-3'; G protein-coupled receptor 65 (GPR65), forward, 5'-GAAATGGCAAATCAACCTCAAC-3' and reverse, 5'-TTCCTTGTTTTCCGTGGCTT-3'; frizzled class receptor 8 (FZD8), forward, 5'-GGAGTGGGGTTACC TGTTGG-3' and reverse 5'-CTTGCGTGTCGTGGTTGAA-3'; SHC adaptor protein 2 (SHC2), forward, 5'-TACGTCGTGCGG TACATGG-3' and reverse, 5'-AAGCGAAGGTTGCTCT TGC-3'; ADP-ribosyltransferase 5 (ART5), forward, 5'-CAG ATTTGGTAATGCCACCCTC-3' and reverse, 5'-CAAAGC CCAGAATGGAATAGC-3'; TRIM2, forward, 5'-AGGACA AAGACGGTGAGCTG-3' and reverse, 5'-CTCTTCACGCCT TCTGTGGT-3'; and GAPDH, forward, 5'-GAGTCAACGGAT TTGGTCGT-3' and reverse, 5'-CCCAGTAGCAGTTCAGG TGG-3'. GAPDH was used as the internal control. The relative expression levels of genes were quantified using the $2^{-\Delta \Delta C q}$ method, as described previously (25). At least three biological repeats and three technical replicates were performed.

Western blot analysis. Total protein from the osteosarcoma cells was lysed and extracted using RIPA lysis buffer and quantified using a BCA Protein Assay kit (both from Beyotime Institute of Biotechnology, Haimen, China) according to the manufacturer's protocol. The protein samples $(40 \mu \mathrm{g})$ were separated by $10 \%$ SDS-PAGE and transferred onto NC membranes (EMD Millipore). The membranes were blocked in 5\% non-fat milk and incubated with the following primary antibodies: TRIM2 (cat. no. ab3942; 1:1,000), matrix metalloproteinase (MMP)9 (cat. no. ab38898; 1:1,000), MMP2 (cat. no. ab37150; 1:500), N-cadherin (cat. no. ab18203; 1:500), E-cadherin (cat. no. ab76055; 1:500), Snail (cat. no. ab180714; 1:1,000), Vimentin (cat. no. ab92547; 1:1,000), protein kinase B (AKT; cat. no. ab8805; 1:500), phosphorylated-AKT (cat. no. ab38449; 1:1,000), protein kinase A (PKA; cat. no. ab75991; 1:2,000), CREB (cat. no. ab31387; 1:1,000), phosphorylatedCREB (cat. no. ab32096; 1:3,000) and anti-GAPDH (cat. no. ab9485; 1:2,000) (all from Abcam) at $4^{\circ} \mathrm{C}$ overnight. The membranes were then incubated with secondary antibodies, anti-rabbit IgG (cat. no. ab205718; 1:2,000), anti-goat IgG (cat. no. ab6566; 1:2,000) or anti-mouse IgG (cat. no. ab6728; $1: 2,000$ ) (all from Abcam) for $2 \mathrm{~h}$ at room temperature. The complexes were detected by ECL (Forevergen Biosciences, Guangzhou, China). GAPDH was applied as the internal standard. Six samples for each group were used for western blot analysis.

In vivo nude mouse models. All animal experiments were approved by the Institute Research Medical Ethics Committee of Sun Yat-Sen University (Guangzhou, China). Male BALB/C nude mice (age, 5-6 weeks; weight, 18-22 g) were obtained from the Shanghai Laboratory Animal Center (Shanghai, China). Animals were maintained in a specific pathogen-free environment throughout the experiments under the following

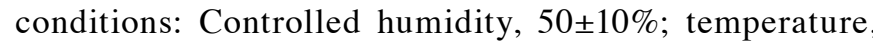
$23 \pm 2^{\circ} \mathrm{C} ; 12$-h light/dark cycle and ad libitum access to food and water. U2OS cells $\left(2 \times 10^{6}\right)$ in a volume of $100 \mu 1$ were subcutaneously injected into the caudal veins of the mice. Post-implantation, five of the mice were sacrificed and the tumors were surgically dissected at 5 days. The weight and size of the cancerous tissues were then examined.
Statistical analysis. Data are presented as the mean \pm standard error of the mean unless otherwise indicated. Significance was established using SPSS PASW Statistics 18.0 (SPSS, Inc., Chicago, IL, USA) software and GraphPad Prism 5 (GraphPad Software, Inc., La Jolla, CA, USA) software. For data with a normal distribution, comparisons were performed using independent t-tests, one-way analysis of variance (ANOVA) and two-way ANOVA, with the LSD-t-test used for the post hoc test. The non-parametric Mann-Whitney U test, K-S test, Kruskal-Wallis test and Wilcoxon test were performed if the data did not have a normal distribution, and the Nemenyi test was used for multiple comparisons. $\mathrm{P}<0.05$ was considered to indicate a statistically significant difference. The significant level was corrected if necessary.

\section{Results}

High expression of TRIM2 in osteosarcoma tissue is correlated with lower survival rates and induction of metastasis. The expression profiles were collected from the NCBI GEO (GSE21257). By combining the expression profiles and the clinical properties, the association between the expression levels of TRIM2, STC2 and VIM and survival rate were analyzed. The results showed that the survival difference was statistically significant between the high and low expression level of TRIM2 and VIM. Regardless of whether overall survival or distal metastasis-free survival rate, a high expression level of TRIM2 and VIM led to a reduced rates of survival and distal metastasis-free survival (Fig. 1A-C). Higher expression levels of TRIM2 and VIM reduced the survival percentage by $50 \%$ at 40 days. However, for STC2, although the survival percentage was reduced when its expression level was high, the difference between a low expression level of STC2 was not significant $(\mathrm{P}>0.05)$. Therefore, the evidence confirmed that a high expression of TRIM2 was correlated with lower survival rate and distal metastasis-free survival rate. By contrast, when the expression level of TRIM2 was compared in primary tumor and metastatic tumor tissues, only the metastatic tissues exhibited a significantly higher level of TRIM2, compared with the primary tumor tissues or in tumor tissues with metastasis within 5 years (Fig. 1D and E). Therefore, actual osteosarcoma tumor tissues and normal tissues were collected for the immunohistochemical staining to determine the expression level of TRIM2. The TRIM2 molecules were stained a brown color specifically. The results showed that the level and density of the TRIM 2 molecules were significantly higher in the osteosarcoma tissues compared with the normal tissues (Fig. 1F). According to the preliminary analysis, it was concluded that TRIM2 in osteosarcoma tissues performs specific functions that lead to lower survival rate and induces metastasis. Therefore, the high expression level of TRIM2 may also be an indicator of osteosarcoma.

TRIM2 performs functions in regulating cell proliferation, migration, invasion and apoptosis. In order to verify the functions of TRIM2, in vitro cell assays were performed. The cell lines were examined for the expression level of TRIM2 in order to identify the appropriate line for the following assays. From the results, the hFOB cell line exhibited the lowest expression signal of TRIM2 among the examined cell lines. In the remaining 
A

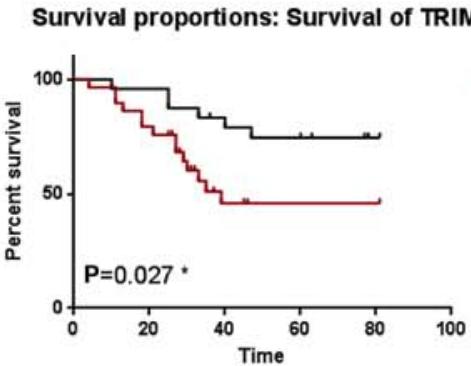

B

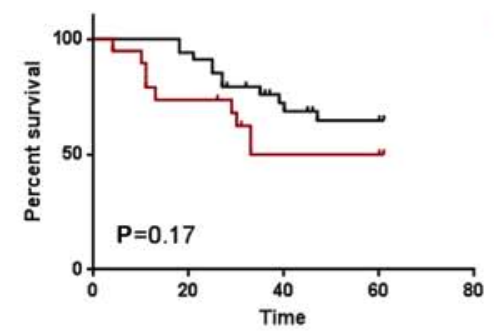

C

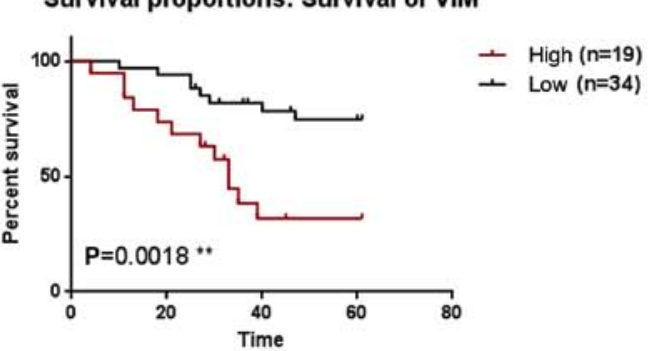

$+\operatorname{High}(n=19)$

- Low $(n=34)$

$\perp$ High $(n=29)$
$\perp \quad$ Low $(n=24)$
Distal metastasis-free survival: Survival of TRIM2

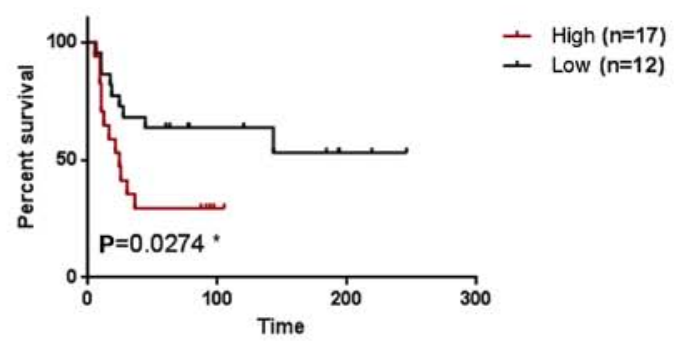

Distal metastasis-free survival: Survival of STC2

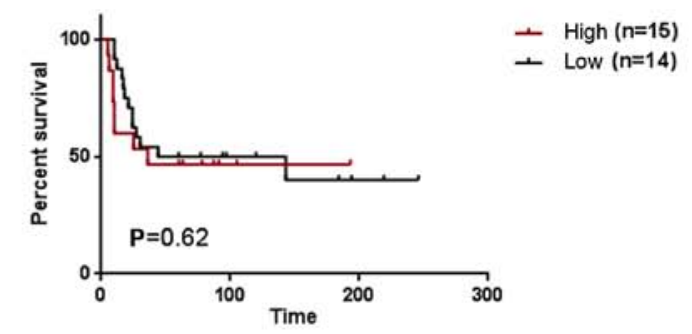

Distal metastasis-free survival: Survival of VIM

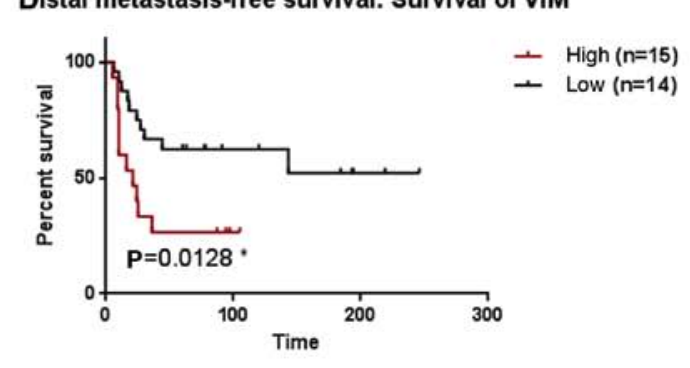

D
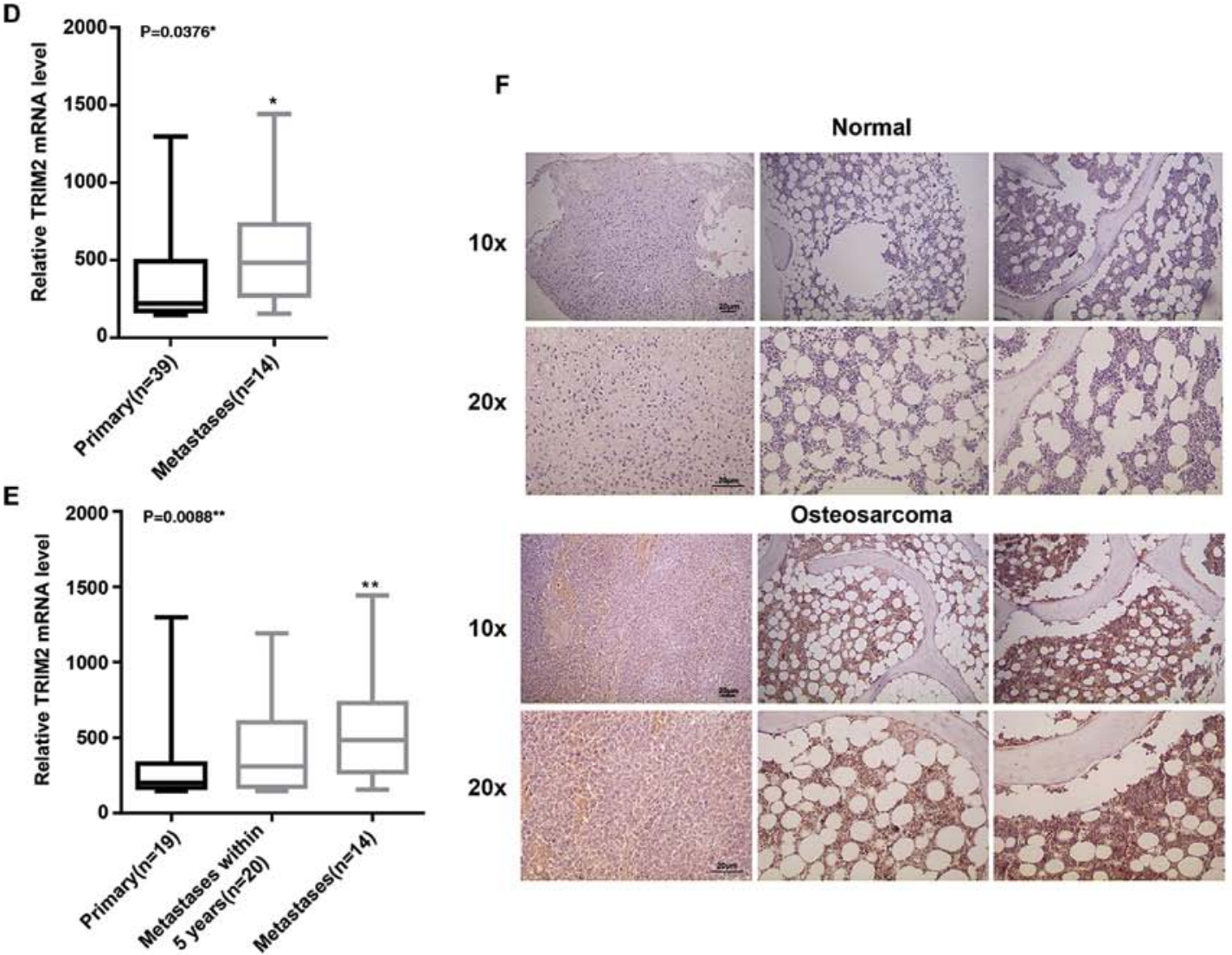

Figure 1. Prediction of the prognosis and metastasis of osteosarcoma by candidate genes. Survival percentage of cells with expression of (A) TRIM2, (B) STC2, and (C) VIM (survival and distal metastasis-free survival). Comparison of relative mRNA level of TRIM2 between primary tumor tissues and (D) metastatic tumor tissues and (E) metastasis within 5 years by combining the expression profiles and the clinical properties. (F) Immunohistochemical staining showing the amounts and density of the TRIM2 molecules in the osteosarcoma and normal tissues. ${ }^{*} \mathrm{P}<0.05$ and ${ }^{* *} \mathrm{P}<0.01$. TRIM2, tripartite motif-containing protein 2 ; STC2 stanniocalcin 2; VIM, vimentin. 
A

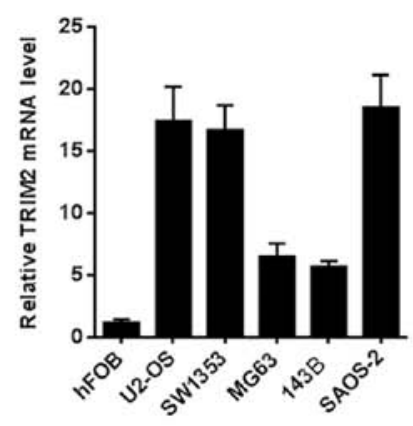

C

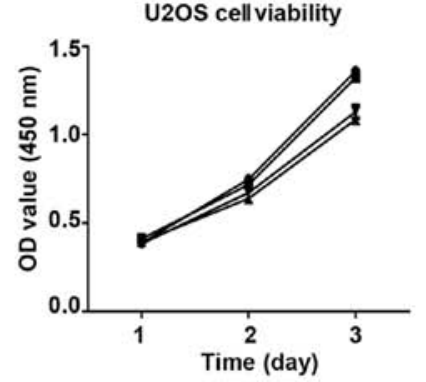

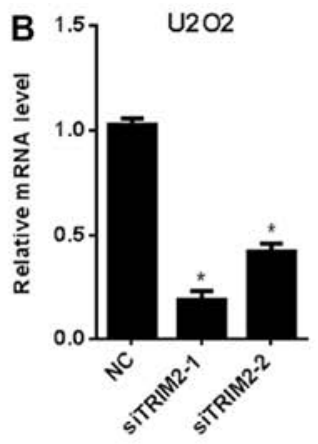

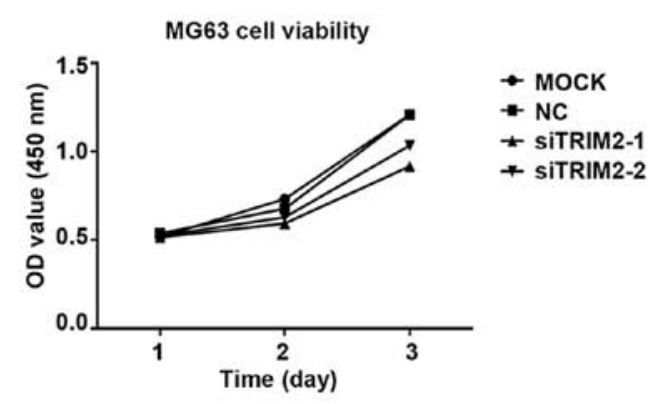

D

U20s
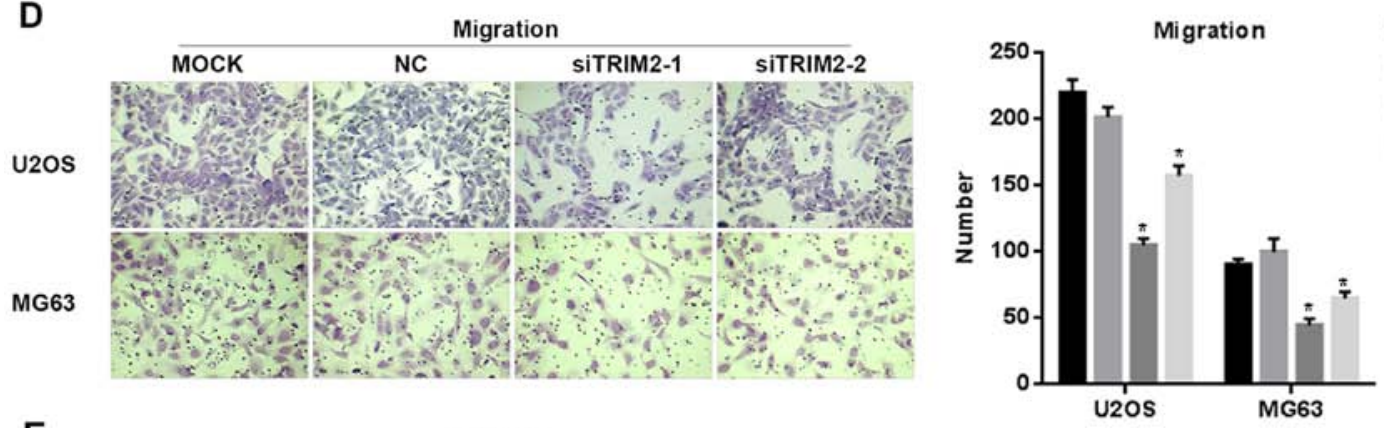

mock

NC

- MOCK

- siTRIM2-1

* siTRIM2-2

E

Invasion
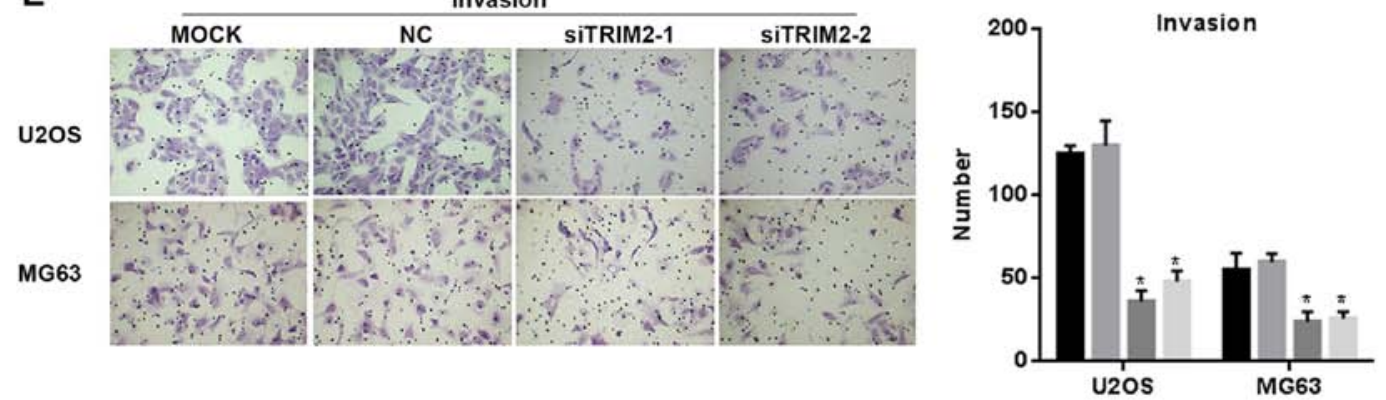

Figure 2. Levels of TRIM2 in cell lines and design of siRNAs targeting TRIM2. (A) In vitro cell assays were performed to examine the expression level of TRIM2 in cell lines. (B) Expression levels of TRIM2 in U2OS and MG63 cells following transfection with TRIM2-targeted siRNAs. (C) Cell viability of U2OS and MG63 cells transfected with siTRIM2-1 or siTRIM2-2, determined via MTS assay. Levels of (D) migrated and (E) invasive cells among groups, determined by immunohistochemistry (original magnification, $\mathrm{x} 200$ ). ${ }^{*} \mathrm{P}<0.05$. siRNA, small interfering RNA. TRIM2, tripartite motif-containing protein 2 ; NC, negative control; OD, optical density.

cell lines, U2OS, SW1353 and SAOS2 cells exhibited relatively high expression levels of TRIM2 among the six cell lines, whereas MG63 and 143B expressed comparatively lower levels of TRIM2 (Fig. 2A). Therefore, U2OS and MG63 were selected for the following assays, which exhibit high and low expression level of TRIM2, respectively. siRNAs targeting TRIM2 were designed. The sequences of the siRNAs were synthesized and integrated into the vector, and the vector was packed into the lentivirus and transfected into U2OS and MG63 cells in order to suppress the expression of TRIM2. The results revealed that siTRIM2-1 and siTRIM2-2 successfully decreased the expression level of TRIM2 (Fig. 2B). Among the two siRNAs,
siTRIM2-1 decreased the level to $\sim 25 \%$ whereas siTRIM2-2 decreased the level to $\sim 50 \%$, compared with the control group, in the U2OS and MG63 cell lines. Cell viability was then determined via an MTS assay. The assay was performed every day for a total of 3 days. The results showed that, for the two cell lines, the viability of the cells transfected with empty vector or without treatment exhibited similar optical density (OD) values at $450 \mathrm{~nm}$. However, for those cells transfected with siTRIM2-1 and siTRIM2-2, the cell viability decreased by $\sim 25 \%$, which was detected on day 3 (Fig. 2C). When the cell migration and invasion capacity of those cells were determined, the migrated cells and invasive cells in the control group and the mock group 


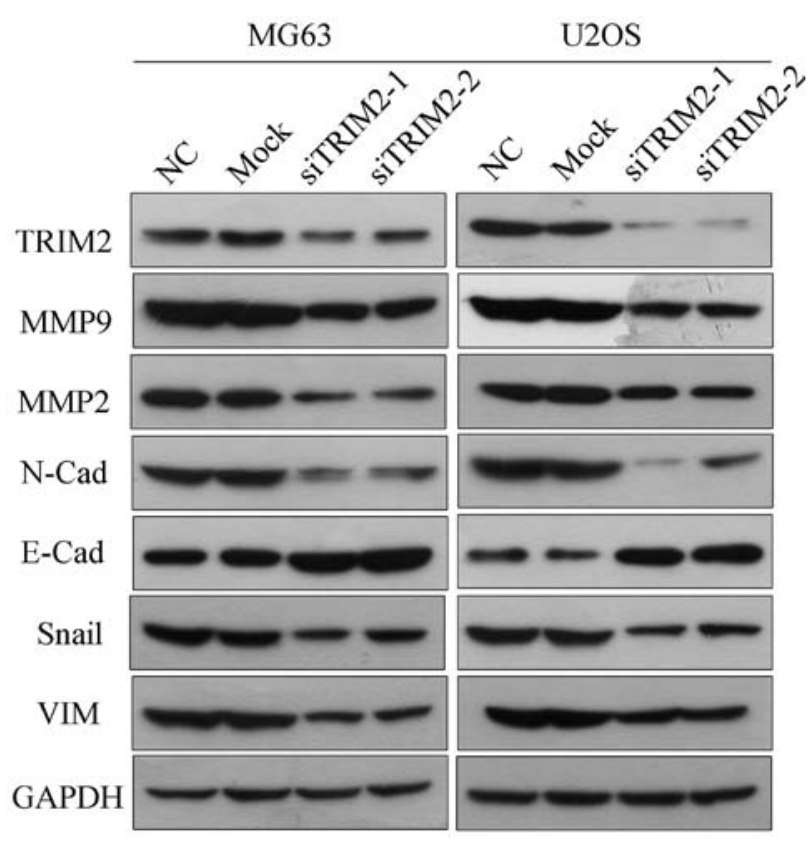

Figure 3. Protein levels of TRIM2 and migration-related genes as detected via western blot analysis. Protein levels of cell migration- and invasion-related molecules, including MMP2, MMP9, N-Cadherin, E-Cadherin, Snail and VIM, were determined by western blot analysis. GAPDH was applied as the internal standard. Six samples were used for each group. TRIM2, tripartite motif-containing protein 2; si, small interfering RNA; NC, negative control; MMP, matrix metalloproteinase; VIM, vimentin.

were increased, with cells exhibiting in higher density in the area; in the siTRIM2-1 and siTRIM2-2 treatment groups, fewer migrated and invasive cells were observed than in the control group and mock group (Fig. 2D and E). This indicated that the migration and invasion capacities of the cells were inhibited when the level of TRIM2 was decreased. Subsequently, the protein from these cells was extracted in order to determine the protein levels of TRIM2 via western blot analysis. According to the results, the protein level of TRIM2 was decreased in the MG63 and U2OS cell lines when transfected the TRIM2-targeted siRNAs (Fig. 3). Therefore, the protein levels of cell migration- and invasion-related molecules, including $\mathrm{N}$-Cadherin, E-Cadherin, Snail and Vimentin, were examined. The results corresponded to those of the cell migration and invasion assays: When the expression of TRIM2 was inhibited, the levels of MMP9, MMP2, N-Cadherin, Snail and Vimentin were accordingly decreased, whereas the protein level of E-Cadherin was increased, indicating weak cell migration and invasion capacities.

Flow cytometry was also performed to determine cell apoptosis. This involved comparing the cells with and without treatment of TRIM2-targeted siRNA transfection. As shown when siTRIM2-1 and siTRIM2-2 inhibited the level of TRIM2 and thereby led to lower cell viability, the cell apoptosis was increased when TRIM2 was decreased in the results (Fig. 4A). The apoptotic percentage increased $\sim 5$-fold in the siRNA-transfected cells compared with the cells in the control group and mock group. In addition, the cell cycle assay demonstrated that the proportion of MG63 and U2OS cells in $\mathrm{S}$ phase was decreased, whereas the proportion in G1 phase was increased post-transfection with siTRIM2-1 and siTRIM2-2 (Fig. 4B). These findings indicated that most of the cells exhibited reduced viability due to the occurrence of cell apoptosis. Based on the evidence above, nude mice were used to perform the tumor formation assay. When the cells were injected into nude mice, the tumors formed gradually. The tumors were collected and compared on day 20 (Fig. 5). The tumor was shown to be marginally smaller in the siTRIM2 group than that in negative control (NC) group. The volume of the tumors were determined every 5 days for a total of 20 days. The difference between the NC group and siTRIM2 group was significant on day 10 , and the size of the tumor in the NC group was $\sim 2$-fold larger than that in the siTRIM2 on day 20. In terms of the weights of the tumor tissues, the tumor weight in the NC group was 2-fold higher than that in the siTRIM2 group, and the difference was statistically significant. According to the immunohistochemical staining of the metastasis-related proteins, the expression of E-Cadherin was upregulated, whereas the expression of Vimentin and $\mathrm{N}$-Cadherin were downregulated expression when TRIM2 was inhibited (Fig. 5D). These results suggested that the tumor metastasis was suppressed when TRIM2 was at a lower level compared with that in the control group. Therefore, according to the results above, TRIM2 appeared to be an activator of cell proliferation, cell migration and cell invasion, thus inducing tumor metastasis.

Verifying the regulation network of TRIM2. In order to further verify the regulation performed by TRIM2 at the molecular level, transcriptome sequencing was performed for the cells transfected with siTRIM2 and empty vector. The criteria for the significant expression was considered as FDR $\leq 0.01$ and $\log 2$ Ratio $\geq 1$. Under these criteria, the significantly differential expressed genes were tagged in red and green (Fig. 6A). The hierarchical clustering for the overview of the genes in the transcriptome sequencing is shown in Fig. 6B. In the graph, the genes with upregulated expression are tagged in red, and those with downregulated expression are tagged in green. According to the results, the effect of the inhibition of TRIM2 was different between the MG63 and U2OS cells. For the MG63 cells, the differentially expressed genes were predominantly upregulated, whereas, the majority of the genes in the U2OS cells were downregulated (Fig. 6B). The genes were then subjected to GO and KEEG pathway analysis. As shown in the plot graphs (Fig. 6C), the genes involved in transcriptome analysis were involved in several cellular components, molecular functions, and biological processes. From the cellular component perspective, 'cell', 'cell part', and 'organelle' were the top three significantly enriched terms. From the molecular function perspective, 'binding' was the top significantly overrepresented term. From the perspective of biological process, 'biological regulation', 'cellular process' and 'metabolic process' were the top three significantly enriched terms. As shown in Fig. 6D, the 'Wnt signaling pathway' and 'MicroRNAs in cancer' were the top enriched term in the MG63 cells, whereas 'Viral carcinogenesis', 'PI3K-Akt signaling pathway', 'Pathways in cancer', and 'cAMP signaling pathway' were the most significantly enriched terms in the U2OS cells.

According to the data from the cell assays, the expression level of TRIM2 differed in the MG63 and U2OS cells, with MG63 cells showing lower expression of TRIM2 and U2OS 
A
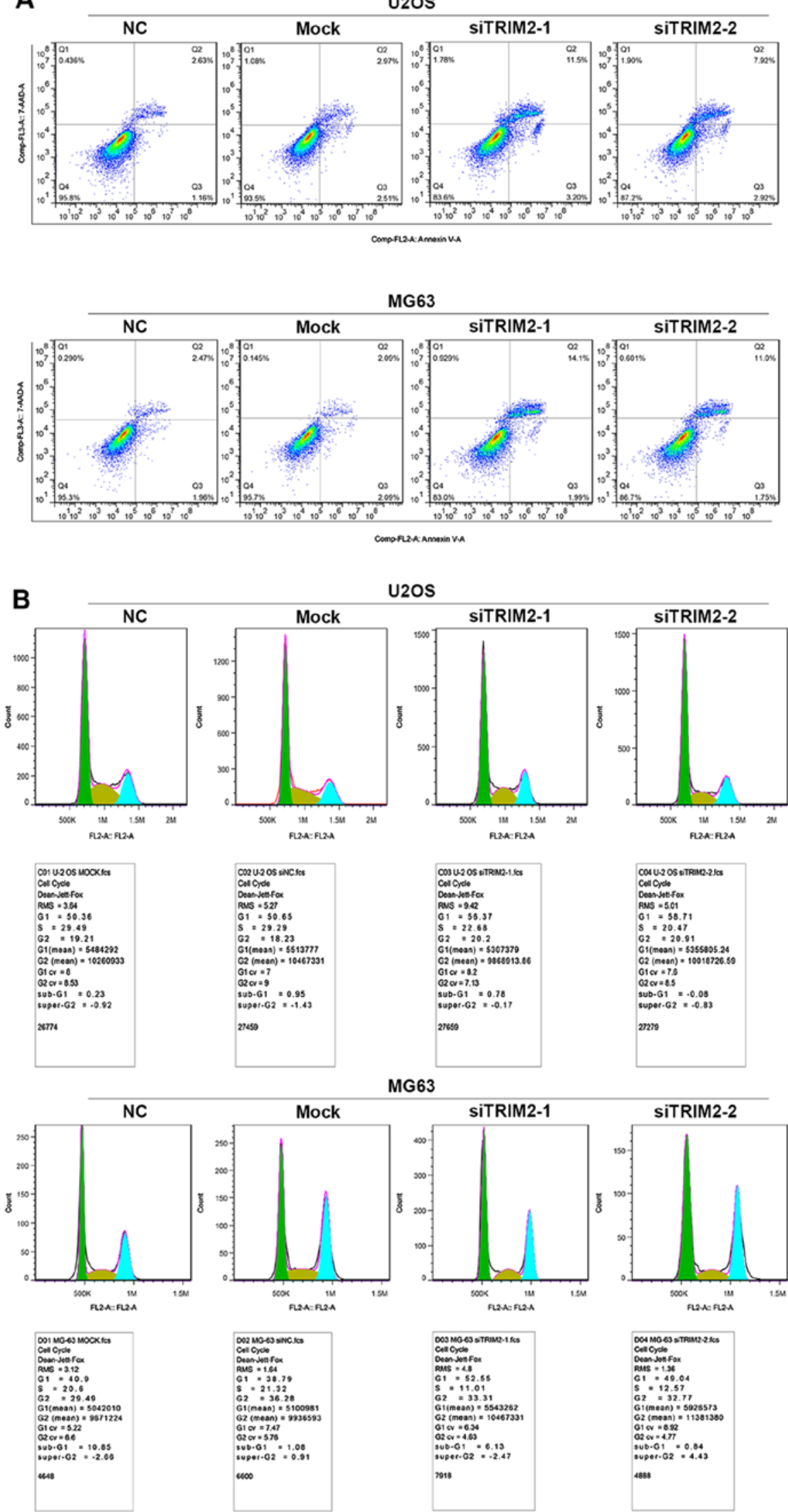

U2OS
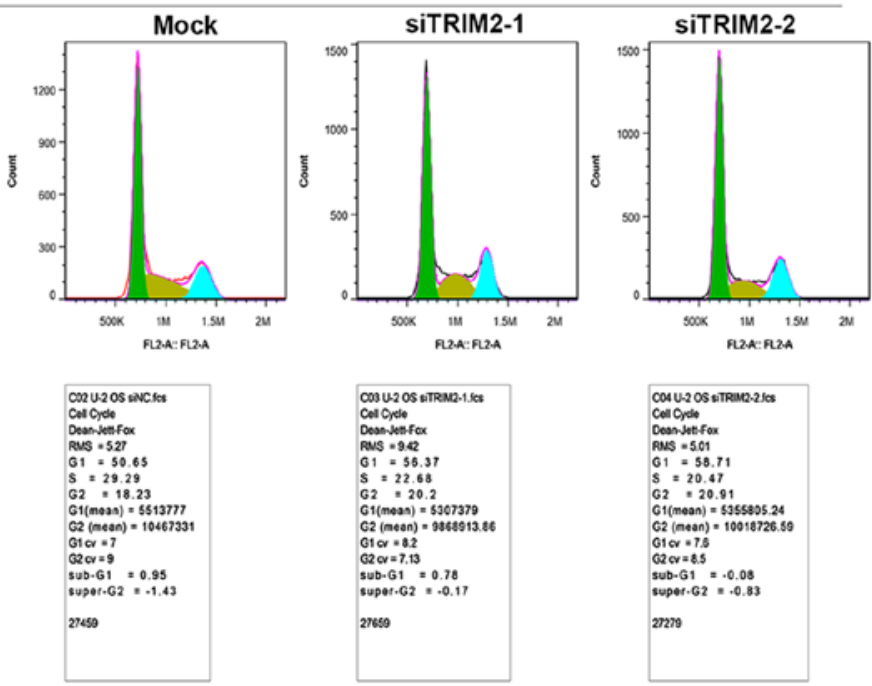

MG63
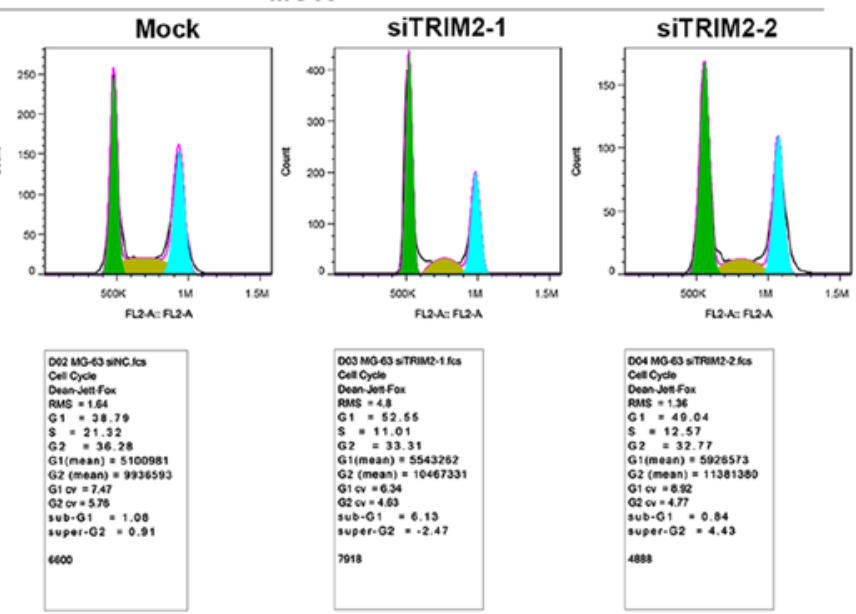

$G 1($ moan $)=5255855.24$
$G 2($ neson $)=1001072659$
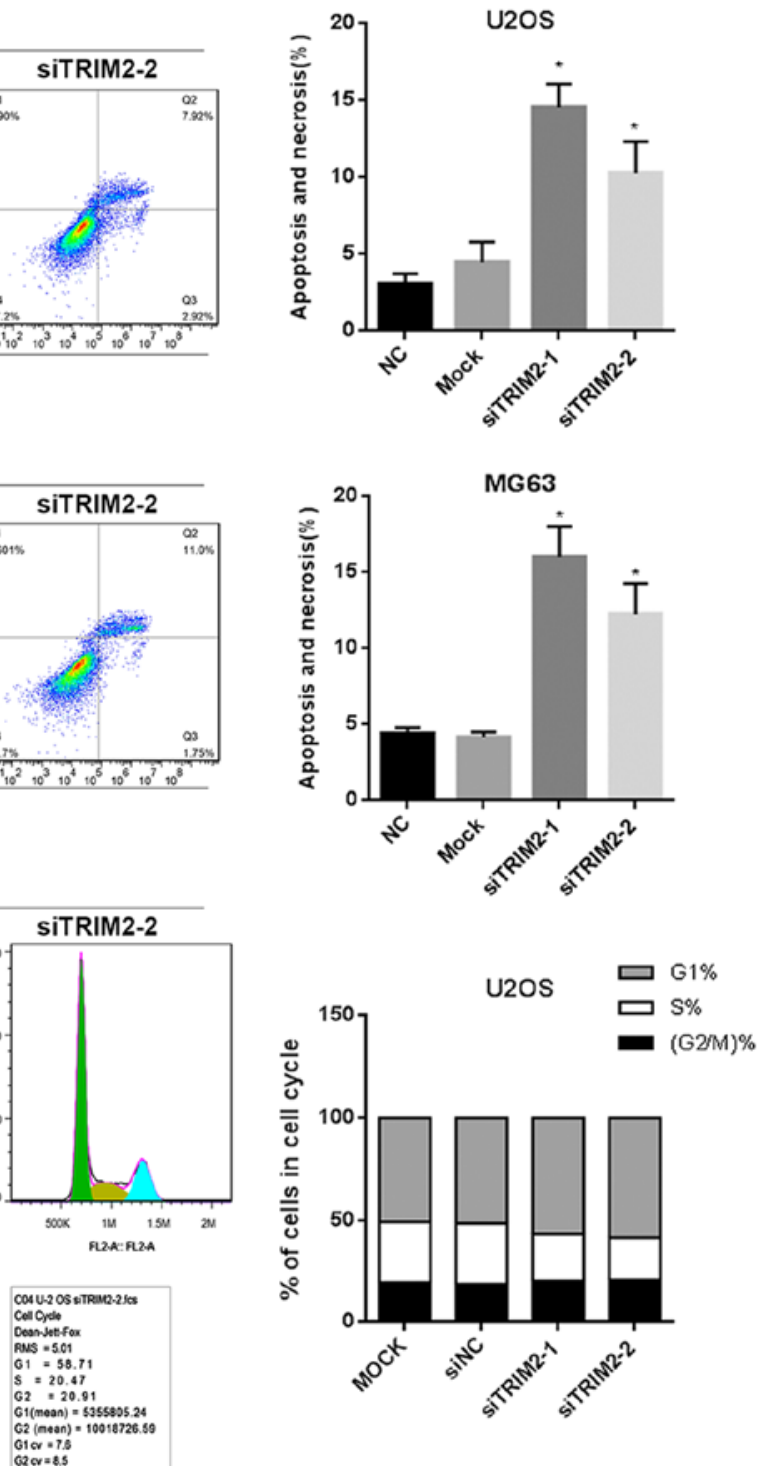

$62 \mathrm{ck}=8.5$
sub-61
super.62

2mo

Figure 4. Cell apoptosis was detected under the regulation of TRIM2 by cell flow cytometry. Flow cytometry was performed to determine (A) cell apoptosis and (B) cell cycle in U2OS and MG63 cells. "P<0.05. TRIM2, tripartite motif-containing protein 2; NC, negative control; si, small interfering RNA.

cells showing higher expression of TRIM2. However, as the two cell lines showed similar results in terms of lower cell viability and capacity to migrate and invade when TRIM2 was inhibited, regulation was expected to be common in these cell lines. 
A

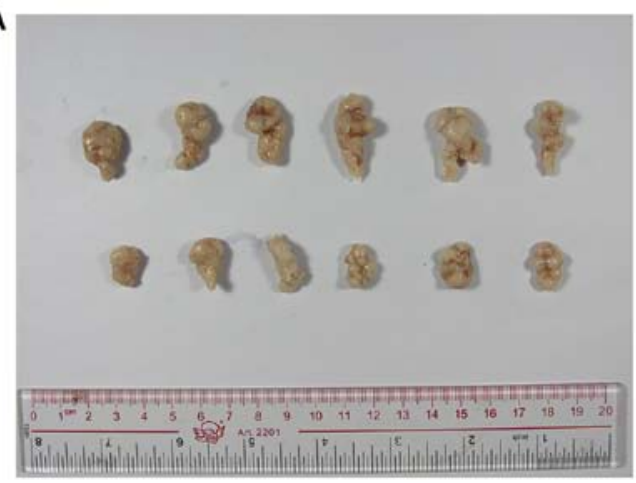

B

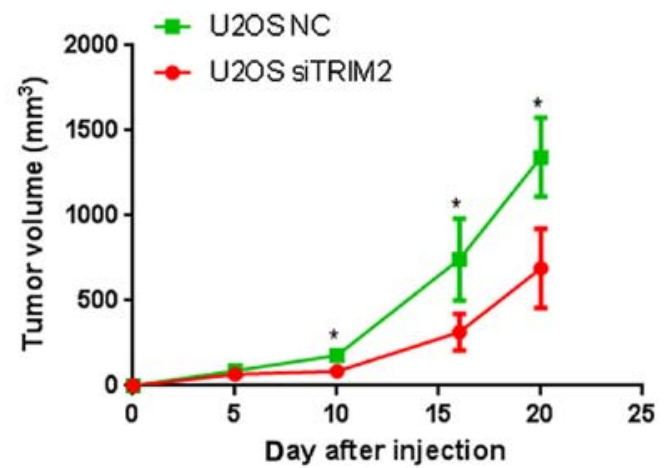

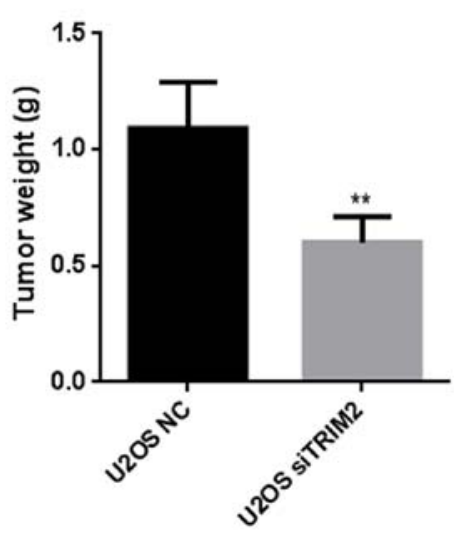

D

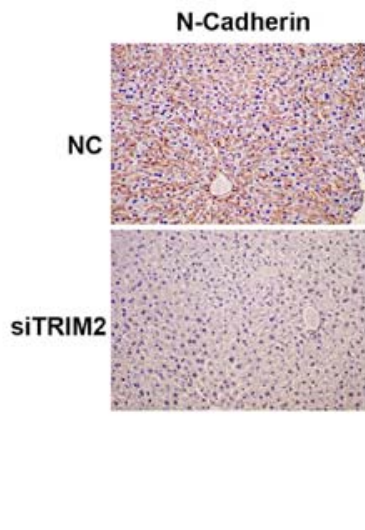

E-Cadherin

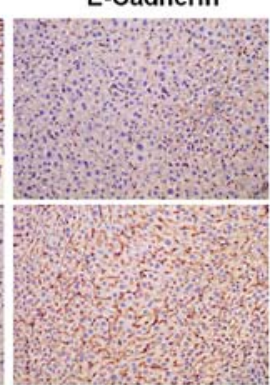

Vimentin

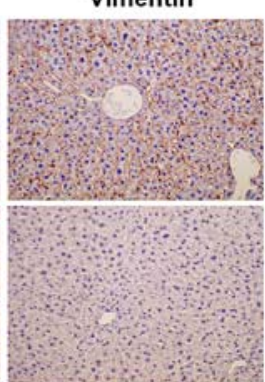

Figure 5. In vivo nude mouse models were used and injected to investigate the regulation of TRIM2 on tumor formation and metastasis. (A) Tumor external dimension, (B) tumor volume and $(\mathrm{C})$ tumor weight were compared between the control and mock groups. ${ }^{*} \mathrm{P}<0.05$ and ${ }^{* * *} \mathrm{P}<0.01$. (D) Metastasis-related proteins were determined by immunohistochemical staining in the NC group and siTRIM2 group (original magnification, x200). Six samples were used for each group for immunohistochemical staining. TRIM2, tripartite motif-containing protein 2; NC, negative control; si, small interfering RNA

Therefore, seven significantly differentially expressed genes related to TRIM2 were extracted from the expression profiles and further validated via RT-qPCR analysis. In the U2OS cells, the TRIM2 gene was significantly downregulated, whereas the remaining genes, with the exception of FZD8 and SHC2, were significantly upregulated in the two cell lines (Fig. 7). For FZD8 and SHC2, they were significantly increased in the MG63 cells but did not in the U2OS cells. These two genes may not be closely associated with TRIM2. As the consistently significantly expressed genes are involved in the regulation of cell cycle, it was hypothesized that TRIM2 performs its functions in osteosarcoma by regulating these genes.

Western blot analysis was used to verify those genes that may involved in the regulatory pathways indicated by the KEEG analysis. The results, as shown in Fig. 8, indicated that, when TRIM2 was inhibited in the U2OS and MG63 cell lines, the protein level of p-AKT was decreased, whereas AKT was not changed. The protein levels of PKA, CREB and phosphorylated CREB were not affected by the inhibition of TRIM2. The results suggested that TRIM2 regulates the development and metastasis of tumorous cells of osteosarcoma via the PI3K/AKT pathway.

\section{Discussion}

TRIM2 was selected and its effects were verified using TRIM2 siRNA in vitro and in vivo in the present study; TRIM2 was shown to be correlated with tumorous cell proliferation, invasion, migration and apoptosis. It may be a novel therapeutic target for the diagnosis and treatment of osteosarcoma. TRIM2 is a member of the TRIM-NHL protein family, which functions as an E3 ubiquitin ligase. It can interact with B-cell lymphoma 2-interacting mediator (Bim) of cell death (26). TRIM2 usually binds to Bim when it is phosphorylated by the p42/p44 mitogen-activated protein kinase (MAPK) pathway. As TRIM2 was expressed at a higher level in patients with osteosarcoma, the level of Bim is expected to be accordingly decreased, thus leading to the enhanced cell proliferation of the tumorous cells $(26,27)$. In previous studies, the association between TRIM2 and osteosarcoma have not been reported. In studies focused on different types of carcinoma, TRIM2 has been detected to be significantly differentially expressed in ovarian cancer, breast cancer, cervical carcinoma, buccal mucosa carcinoma and follicular carcinoma (27-32). In a study of ovarian cancer, the expression of TRIM2 was detected as upregulated, and negatively regulated Bim (7). This finding is consistent with the results of the present study. However, certain studies have shown the opposite results, with the expression of TRIM2 found to be downregulated $(28,31,32)$. The bias between different types of cancer suggests that TRIM2 is an important gene involved in the pathogenic process of cancer, although the underlying mechanism may be different and complex. However, the dysfunction of TRIM2 may be one of the factors involved in the occurrence and development of osteosarcoma to a certain extent. 
A

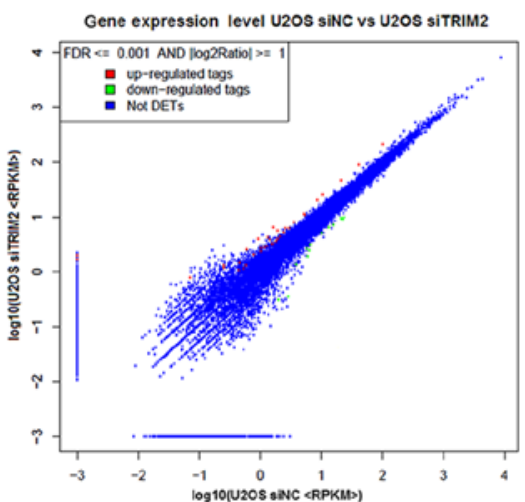

B

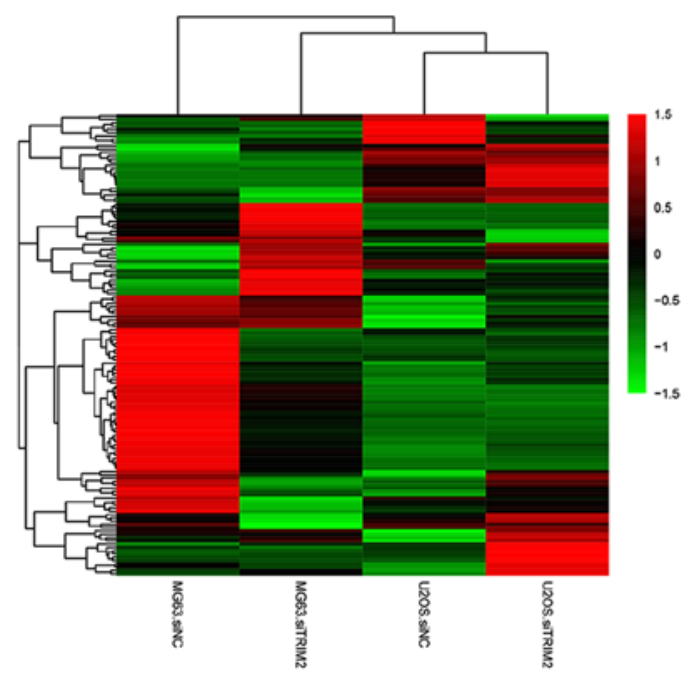

D

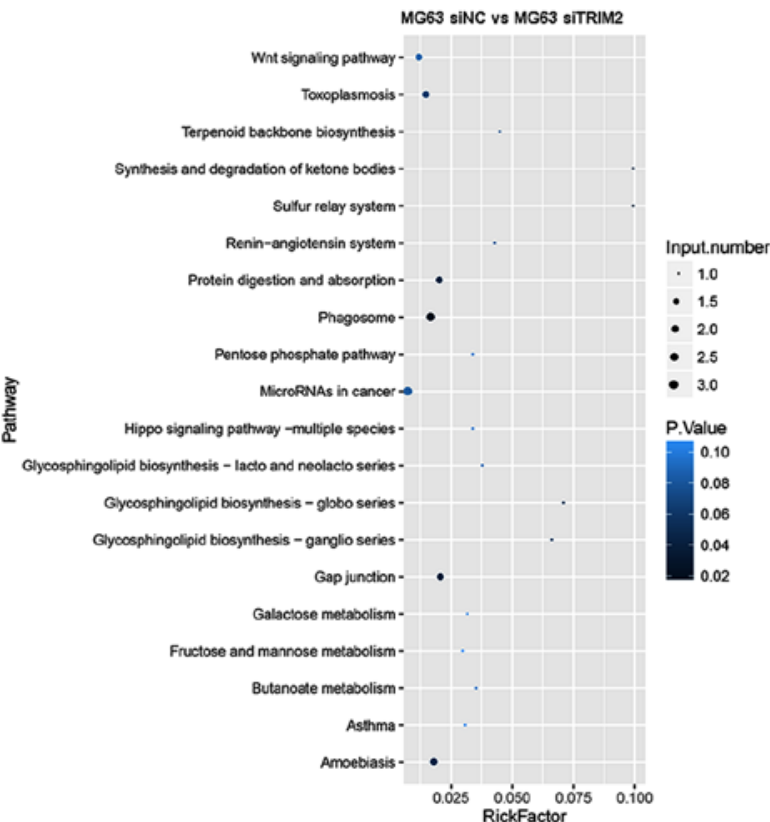

C
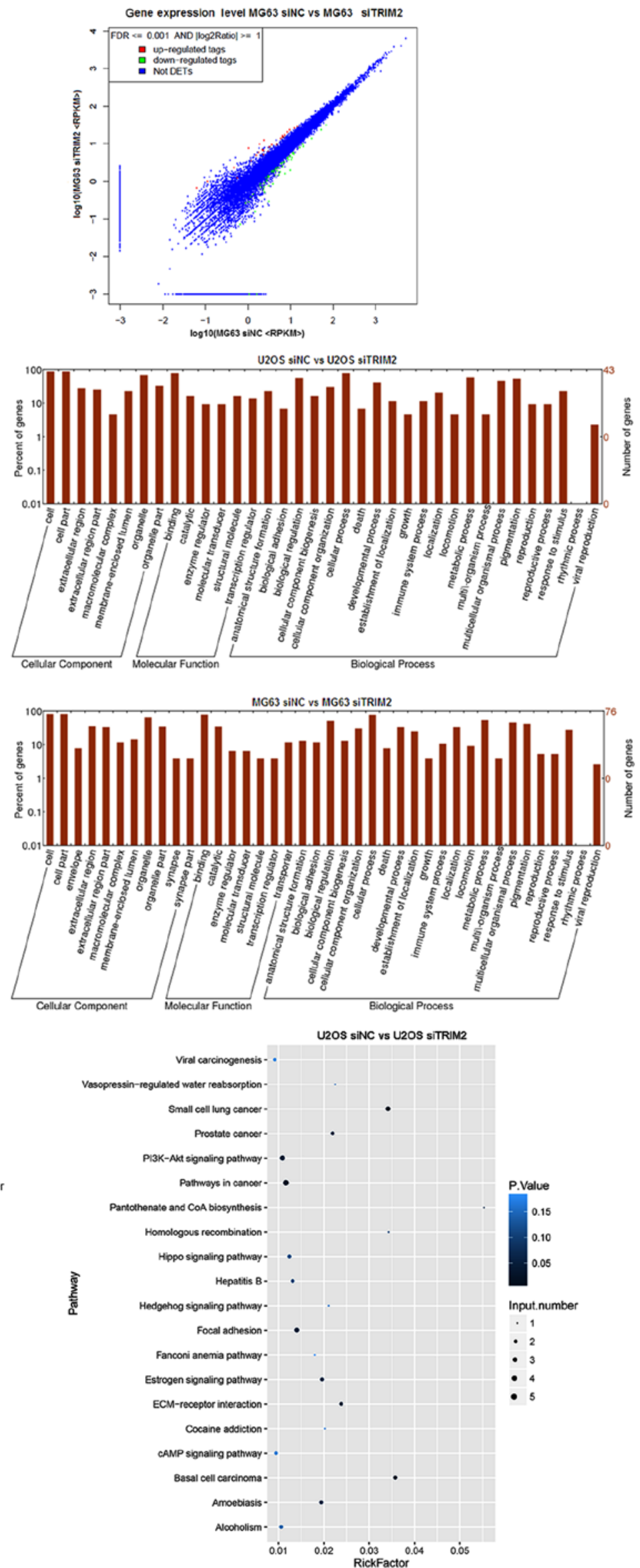

Figure 6. Expression profile and GO analysis of the MG63 and U2OS cells. (A) Hierarchical clustering for the overview of the genes in transcriptome sequencing. (B) Visualization of significantly differentially expressed genes of the MG63 and U2OS cells using a scatter plot. (C) GO analysis and (D) pathways of the MG63 and U2OS cells. GO, Gene Ontology; si, small interfering RNA; NC, negative control; TRIM2, tripartite motif-containing protein 2. 
A

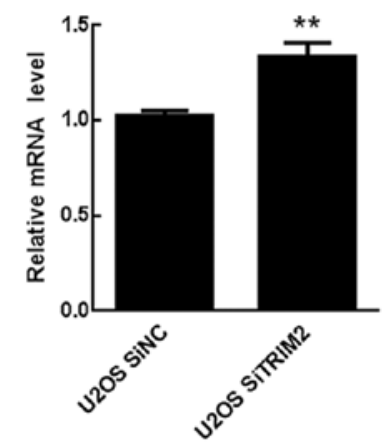

FZD8

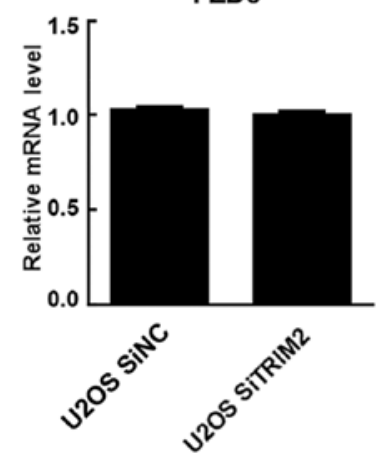

B

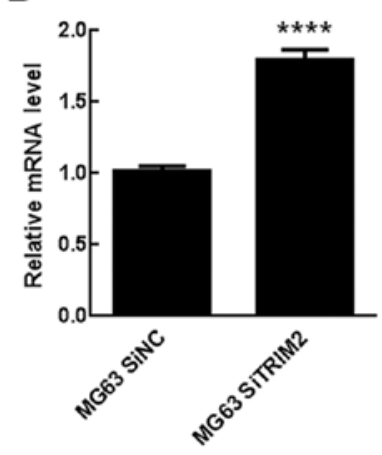

FZD8

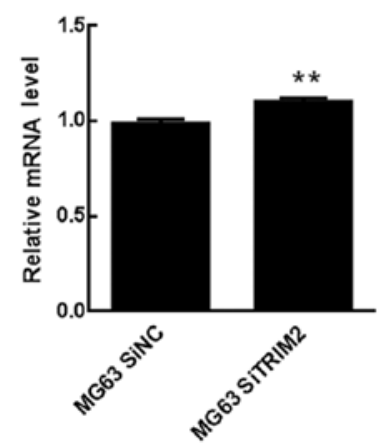

CREB5

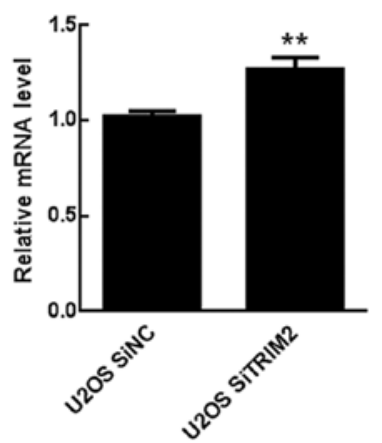

SHC2

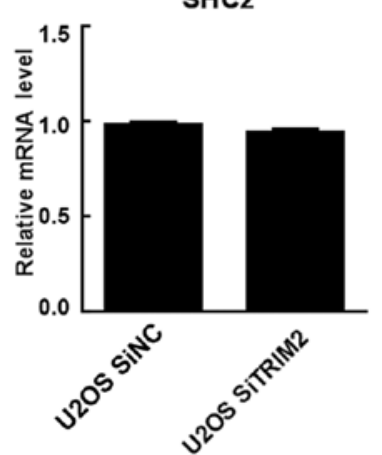

CREB5

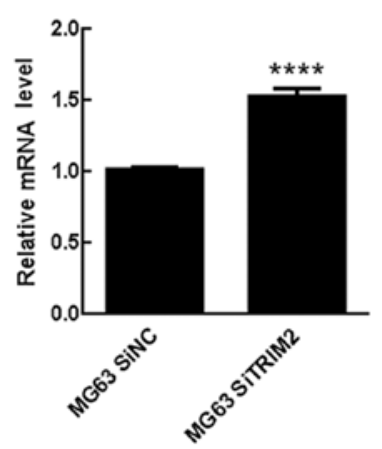

SHC2

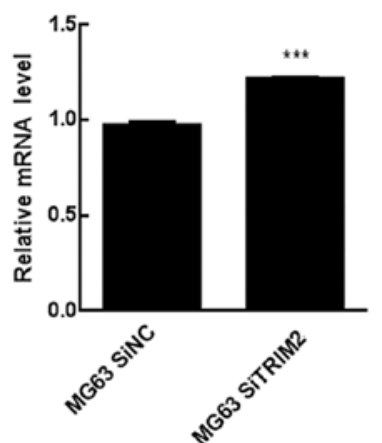

SIRT4

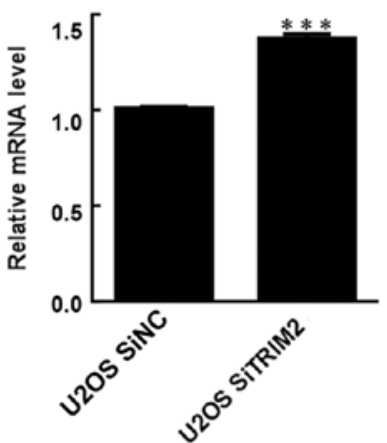

ART5

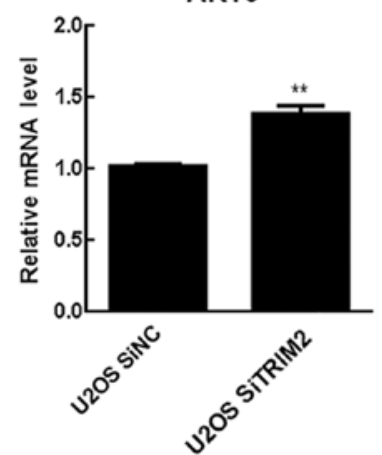

SIRT4

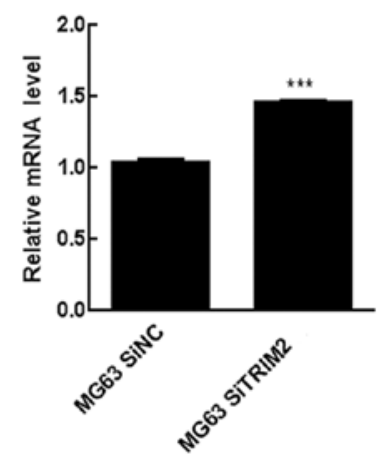

ART5

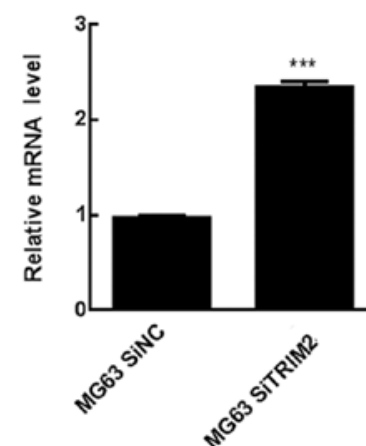

GPR65

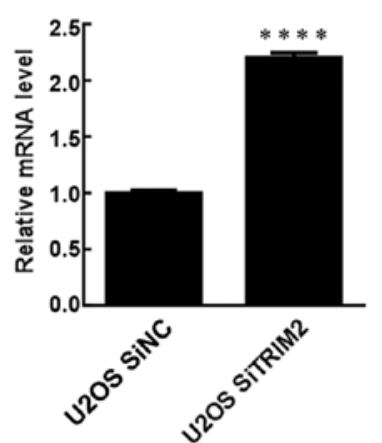

TRIM2

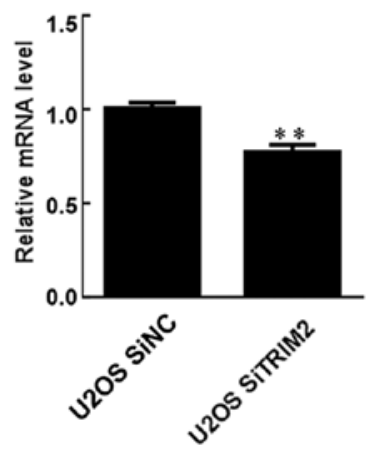

GPR65

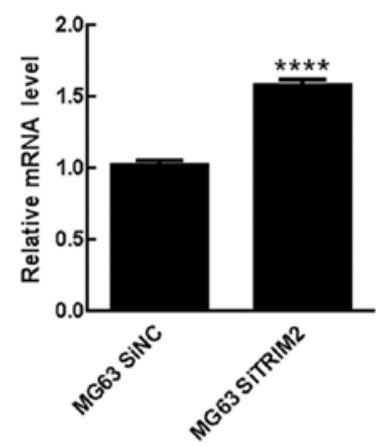

TRIM2

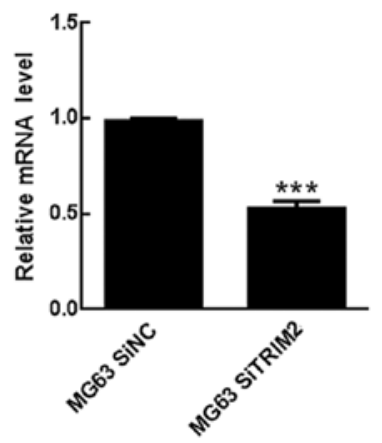

Figure 7. Genes involved in the regulation network of TRIM2. Relative mRNA level of genes associated with TRIM2 were detected in (A) U20S and (B) MG63 cells by reverse transcription-quantitative polymerase chain reaction analysis. ${ }^{* *} \mathrm{P}<0.01,{ }^{* * *} \mathrm{P}<0.001$ and ${ }^{* * * *} \mathrm{P}<0.0001$. ART5, ADP-ribosyltransferase 5 ; CREB5, cAMP responsive element binding protein 5; DDIT3, DNA damage-inducible transcript 3; FZD8, frizzled class receptor 8; GPR65, G protein-coupled receptor 65; NC, negative control; SHC2, SHC adaptor protein 2; si, small interfering RNA; SIRT4, Sirtuin 4; TRIM2, tripartite motif-containing protein 2.

The epithelial-mesenchymal transition (EMT) is a process whereby epithelial cells transition into cells of the mesenchymal phenotype, and it has long been known to be involved in cellular differentiation during development and tumor invasion. EMT results in cells with migratory and invasive properties, the mesenchymal state is associated 


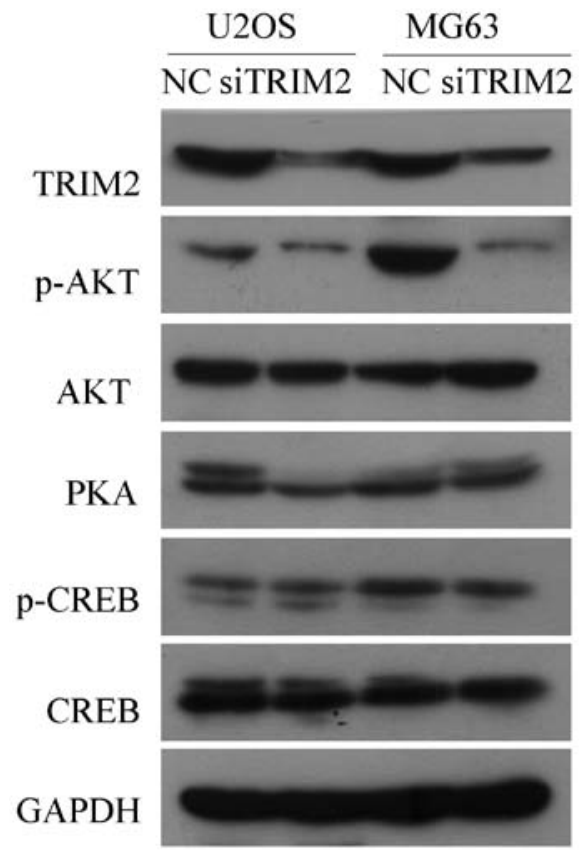

Figure 8 . Western blot analysis to verify genes that may be involved in the regulating pathways of TRIM2. Protein levels of the genes that may be involved in the regulating pathways of TRIM2, including p-AKT, AKT, PKA, p-CREB and CREB, were detected by western blot analysis. GAPDH was applied as the internal standard. Six samples were used for each group. $\mathrm{AKT}$, protein kinase $\mathrm{B}$; CREB, cAMP response element binding protein; $\mathrm{NC}$, negative control; p-, phosphorylated; PKA, protein kinase A; si, small interfering RNA; TRIM2, tripartite motif-containing protein 2.

with the capacity of cells to migrate to distant organs and maintain stemness, enabling their subsequent differentiation into multiple cell types during development and the initiation of metastasis (33). EMT can be induced by various factors. Transforming growth factor- $\beta$ is a major inducer of EMT, it downregulates genes expressed in epithelial cells, including E-cadherin, and upregulates genes normally expressed in mesenchymal cells, including $\mathrm{N}$-cadherin and vimentin $(34,35)$. The degradation of extracellular matrix (ECM) through activating MMPs is an essential step in tumor cell migration. The expression of MMP-2 and MMP-9 is reported to be closely linked with tumor progression and metastasis (36). In the present study, the protein levels of the above-mentioned genes, which are associated with cancer cell development and metastasis, were examined in vitro and in vivo. The results indicated that the inhibition of TRIM2 led to decreased cell migration and invasion capacities, which confirmed that TRIM2 is important in regulating tumorous cell proliferation, migration and invasion.

To investigate the underlying regulatory mechanism of TRIM2, the gene expression was inhibited in expression and screened by RNA-seq for candidate genes. There are few reports on the association between TRIM2 and the candidate genes screened in the present study. Among these genes, SIRT4 and CREB5 are reported to be functionally correlated with tumor development. SIRT4, it is a member of the sirtuin family of $\mathrm{NAD}^{+}$-dependent enzymes, which have various roles in metabolism, stress response and longevity $(37,38)$. It has been reported that SIRT4 negatively regulates mitochondrial glutamine metabolism via the inhibition of glutamate dehydrogenase and has tumor-suppressive functions (39). The decreased level of SIRT4 can lead to poor prognosis in breast cancer (40), and the increased level of SIRT4 contributed to tumorous cell proliferation when TRIM2 was inhibited in the present study. It has been reported that CREB5 may be key in the metastatic signal network of colorectal cancer. A previous report confirmed that metastasis was promoted when CREB5 was expressed at a high level (41). In this study, CREB5 was increased when TRIM2 was downregulated. Therefore, the increase of CREB5 may enhance the metastatic process that leads to inactive cell migration and invasion. However, migrated and invasive cells in the present study were decreased in number when TRIM2 was suppressed. This suggests other genes are likely to regulate the events, rather than CREB5 alone.

For the remaining three genes, ART5, DDIT3 and GPR65, their expression was upregulated when TRIM2 was inhibited. Reports on the functions of these genes in tumor occurrence and development are limited. In analyzing the gene functions independently, ART5 can interact with TIM23. It has been reported that the overexpression of ART5 suppresses TIM23, and defects in cell growth were observed (42). This also explains the decreased cell viability in the TRIM2 inhibition group in the present study. DDIT3 is able to function in inducing DNA damage. The overexpression of DDIT3 can damage cell migration and invasion according to a previous study $(43,44)$. Therefore, it may be an important gene contributing to the decreased cell migration and invasion observed when TRIM2 was suppressed. GPR65, is a gene involved in the p38 MAPK signaling pathway, which is correlated with the cell cycle (45). However, its exact function, particularly involving tumor development, remains to be fully elucidated.

The results from KEGG analysis indicated that 'Wnt signaling pathway' and 'MicroRNAs in cancer' were the top enriched terms in MG63 cells, and 'Pathways in viral carcinogenesis', 'PI3K-Akt signaling pathway', 'Pathways in cancer', and 'cAMP signaling pathway' were the most significantly enriched terms in U2OS cells. The present study mainly focused on PI3K/AKT and cAMP signaling pathways and examined whether TRIM2 was implicated in these regulating pathways. A number of studies have shown that the PI3K/AKT pathway is involved in osteosarcoma (46-48). mTOR, one of the PI3K family members, is important in the regulation of cell cycle, growth and development; the PI3K/AKT/mTOR signaling pathway is reported to be involved in the proliferation, migration and survival of osteosarcoma (49). The results of the present study showed that, in the U2OS and MG63 cell lines, the expression of TRIM2 was inhibited, the protein level of phosphorylated-AKT was decreased, and the level of AKT was unchanged, which indicated that the AKT signaling was implicated in the regulatory pathway of TRIM2. A previous study demonstrated that the CAMP/PKA signaling pathway was involved in osteosarcoma chemoresistance (50). Parathyroid hormone (PTH) stimulates bone formation in animals and humans. Studies have indicated that the cAMP/PKA pathway is involved in PTH signaling transduction, which subsequently affects the development of osteosarcoma cells $(51,52)$. The present study failed to show evidence that the cAMP/PKA pathway was implicated in the development of osteosarcoma, which may due to the small sample size, and further 
investigation is required to confirm the results. Taken together, the results suggested that TRIM2 regulated the development and metastasis of osteosarcoma tumor cells via the PI3K/AKT pathway.

In conclusion, the present study determined the functions of TRIM2 in osteosarcoma cell lines. TRIM2 is important in regulating the tumorous cell proliferation, migration and invasion. The SIRT4, ART5 and DDIT3 genes appear to be functionally correlated with TRIM2, which regulates the pathogenic processes of osteosarcoma, and the PI3K/AKT signaling pathway may be involved in the regulatory role of TRIM2 in the development and metastasis of osteosarcoma, however, comprehensive assays are required to validate this. The importance of TRIM2 requires further validation in clinical samples, and the comprehensive analysis of clinical samples is likely to benefit the development of diagnosis and treatment for osteosarcoma.

\section{Acknowledgements}

Not applicable.

\section{Funding}

This study was supported by the Science and Technology Planning Project of Guangdong Province, China (grant nos. 2016A020225001 and 2017A020213024), the Administration of Traditional Chinese Medicine of Guangdong Province, China (grant no. 20161233) and the Medical Scientific Research Foundation of Guangdong Province, China (grant no. A2016233).

\section{Availability of data and materials}

The datasets used and/or analyzed during the current study are available from the corresponding author on reasonable request.

\section{Authors' contributions}

YQ and JY conceived and designed the study, and critically revised the manuscript. JY performed the experiments, analyzed the data and drafted the manuscript. FZ, SH and SW was involved in study design, study implementation and manuscript revision. All authors have read and approved the final manuscript.

\section{Ethics approval and consent to participate}

The present study was approved by the Institutional Research Ethics Committee of Jinan University. All animal experiments were approved by The Institute Research Medical Ethics Committee of Sun Yat-Sen University. Informed consent was provided by all participants prior to the study.

\section{Patient consent for publication}

Not applicable.

\section{Competing interests}

The authors declare that they have no competing interests.

\section{References}

1. Kashima T, Nakamura K, Kawaguchi J, Takanashi M, Ishida T, Aburatani H, Kudo A, Fukayama $M$ and Grigoriadis AE: Overexpression of cadherins suppresses pulmonary metastasis of osteosarcoma in vivo. Int J Cancer 104: 147-154, 2003.

2. Steeg PS: Tumor metastasis: Mechanistic insights and clinical challenges. Nat Med 12: 895-904, 2006.

3. Bacci G, Rocca M, Salone M, Balladelli A, Ferrari S, Palmerini E, Forni $\mathrm{C}$ and Briccoli A: High grade osteosarcoma of the extremities with lung metastases at presentation: Treatment with neoadjuvant chemotherapy and simultaneous resection of primary and metastatic lesions. J Surg Oncol 98: 415-420, 2008.

4. Harting MT, Blakely ML, Jaffe N, Cox CS Jr, Hayes-Jordan A, Benjamin RS, Raymond AK, Andrassy RJ and Lally KP: Long-term survival after aggressive resection of pulmonary metastases among children and adolescents with osteosarcoma. J Pediatr Surg 41: 194-199, 2006.

5. Tsuchiya H, Kanazawa Y, Abdel-Wanis ME, Asada N, Abe S, Isu K, Sugita T and Tomita K: Effect of timing of pulmonary metastases identification on prognosis of patients with osteosarcoma: The Japanese Musculoskeletal Oncology Group study. J Clin Oncol 20: 3470-3477, 2002.

6. Wu PK, Chen WM, Chen CF, Lee OK, Haung CK and Chen TH: Primary osteogenic sarcoma with pulmonary metastasis: Clinical results and prognostic factors in 91 patients. Jpn J Clin Oncol 39: 514-522, 2009.

7. Tsuchiya $\mathrm{H}$, Tomita $\mathrm{K}$, Mori $\mathrm{Y}$, Asada $\mathrm{N}$ and Yamamoto $\mathrm{N}$ : Marginal excision for osteosarcoma with caffeine assisted chemotherapy. Clin Orthop Relat Res 358: 27-35, 1999.

8. Zanotti S and Canalis E: Notch signaling and the skeleton. Endocr Rev 37: 223-253, 2016.

9. Kofler NM, Shawber CJ, Kangsamaksin T, Reed HO, Galatioto J and Kitajewski J: Notch signaling in developmental and tumor angiogenesis. Genes Cancer 2: 1106-1116, 2011.

10. Engin F, Bertin T, Ma O, Jiang MM, Wang L, Sutton RE, Donehower LA and Lee B: Notch signaling contributes to the pathogenesis of human osteosarcomas. Hum Mol Genet 18: 1464-1470, 2009.

11. Tanaka M, Setoguchi T, Hirotsu M, Gao H, Sasaki H, Matsunoshita Y and Komiya S: Inhibition of Notch pathway prevents osteosarcoma growth by cell cycle regulation. Br J Cancer 100: 1957-1965, 2009.

12. Dailey DD, Anfinsen KP, Pfaff LE, Ehrhart EJ, Charles JB, Bønsdorff TB, Thamm DH, Powers BE, Jonasdottir TJ and Duval DL: HES1, a target of Notch signaling, is elevated in canine osteosarcoma, but reduced in the most aggressive tumors. BMC Vet Res 9: 130, 2013.

13. Komori T: Regulation of osteoblast differentiation by transcription factors. J Cell Biochem 99: 1233-1239, 2006.

14. Hilton MJ, Tu X, Wu X, Bai S, Zhao H, Kobayashi T, Kronenberg HM, Teitelbaum SL, Ross FP, Kopan R, et al: Notch signaling maintains bone marrow mesenchymal progenitors by suppressing osteoblast differentiation. Nat Med 14: 306-314, 2008.

15. McManus MM, Weiss KR and Hughes DP: Understanding the role of Notch in osteosarcoma. Adv Exp Med Biol 804: 67-92, 2014.

16. Krishnan V, Bryant HU and Macdougald OA: Regulation of bone mass by Wnt signaling. J Clin Invest 116: 1202-1209, 2006.

17. Cai Y, Cai T and Chen Y: Wnt pathway in osteosarcoma, from oncogenic to therapeutic. J Cell Biochem 115: 625-631, 2014.

18. Hoang BH, Kubo T, Healey JH, Sowers R, Mazza B, Yang R, Huvos AG, Meyers PA and Gorlick R: Expression of LDL receptor-related protein 5 (LRP5) as a novel marker for disease progression in high-grade osteosarcoma. Int J Cancer 109: 106-111, 2004

19. Ma Y,Ren Y,HanEQ,LiH,Chen D,Jacobs JJ, Gitelis S, O'Keefe RJ, Konttinen YT, Yin G, et al: Inhibition of the Wnt- $\beta$-catenin and Notch signaling pathways sensitizes osteosarcoma cells to chemotherapy. Biochem Biophys Res Commun 431: 274-279, 2013.

20. Mödder UI, Oursler MJ, Khosla S and Monroe DG: Wnt10b activates the Wnt, notch, and NFKB pathways in U2OS osteosarcoma cells. J Cell Biochem 112: 1392-1402, 2011.

21. Laplante M and Sabatini DM: mTOR signaling in growth control and disease. Cell 149: 274-293, 2012.

22. Hu K, Dai HB and Qiu ZL: mTOR signaling in osteosarcoma: Oncogenesis and therapeutic aspects (Review). Oncol Rep 36: 1219-1225, 2016. 
23. Perry JA, Kiezun A, Tonzi P, Van Allen EM, Carter SL, Baca SC, Cowley GS, Bhatt AS, Rheinbay E, Pedamallu CS, et al: Complementary genomic approaches highlight the PI3K/mTOR pathway as a common vulnerability in osteosarcoma. Proc Natl Acad Sci USA 111: E5564-E5573, 2014

24. Wang Z, Gerstein M and Snyder M: RNA-Seq: A revolutionary tool for transcriptomics. Nat Rev Genet 10: 57-63, 2009.

25. Livak KJ and Schmittgen TD: Analysis of relative gene expression data using real-time quantitative PCR and the 2(-Delta Delta C(T)) method. Methods 25: 402-408, 2001.

26. Thompson S, Pearson AN, Ashley MD, Jessick V, Murphy BM, Gafken P, Henshall DC, Morris KT, Simon RP and Meller R: Identification of a novel Bcl-2-interacting mediator of cell death (Bim) E3 ligase, tripartite motif-containing protein 2 (TRIM2), and its role in rapid ischemic tolerance-induced neuroprotection. J Biol Chem 286: 19331-19339, 2011.

27. Chen X, Dong C, Law PT, Chan MT, Su Z, Wang S, Wu WK and $\mathrm{Xu} \mathrm{H}$ : MicroRNA-145 targets TRIM2 and exerts tumorsuppressing functions in epithelial ovarian cancer. Gynecol Oncol 139: 513-519, 2015.

28. Williams MD, Zhang L, Elliott DD, Perrier ND, Lozano G, Clayman GL and El-Naggar AK: Differential gene expression profiling of aggressive and nonaggressive follicular carcinomas. Hum Pathol 42: 1213-1220, 2011.

29. Panaccione A, Guo Y, Yarbrough WG and Ivanov SV: Expression profiling of clinical specimens supports the existence of neural progenitor-like stem cells in basal breast cancers. Clin Breast Cancer 17: 298-306.e7, 2017.

30. Ivanov SV, Panaccione A, Nonaka D, Prasad ML, Boyd KL, Brown B, Guo Y, Sewell A and Yarbrough WG: Diagnostic SOX10 gene signatures in salivary adenoid cystic and breast basal-like carcinomas. Br J Cancer 109: 444-451, 2013.

31. Yang K, Zhang G, Mei J, Chen D and Wu M: Screening and analysis of pathogenic genes during DMBA-induced buccal mucosa carcinogenesis in golden hamsters. Oncol Rep 23: $1619-1624,2010$

32. Miyatake T, Ueda Y, Nakashima R, Yoshino K, Kimura T, Murata T, Nomura T, Fujita M, Buzard GS and Enomoto T: Down-regulation of insulin-like growth factor binding protein-5 (IGFBP-5): Novel marker for cervical carcinogenesis. Int J Cancer 120: 2068-2077, 2007.

33. Thiery JP, Acloque H, Huang RY and Nieto MA: Epithelialmesenchymal transitions in development and disease. Cell 139: 871-890, 2009

34. Willis BC,Liebler JM,Luby-Phelps K, Nicholson AG,CrandallED du Bois RM and Borok Z: Induction of epithelial-mesenchymal transition in alveolar epithelial cells by transforming growth factor-beta1: Potential role in idiopathic pulmonary fibrosis. Am J Pathol 166: 1321-1332, 2005.

35. Willis BC and Borok Z: TGF-beta-induced EMT: Mechanisms and implications for fibrotic lung disease. Am J Physiol Lung Cell Mol Physiol 293: L525-L534, 2007.

36. Moses MA, Wiederschain D, Loughlin KR, Zurakowski D, Lamb CC and Freeman MR: Increased incidence of matrix metalloproteinases in urine of cancer patients. Cancer Res 58: $1395-1399,1998$

37. Finkel T, Deng CX and Mostoslavsky R: Recent progress in the biology and physiology of sirtuins. Nature 460: 587-591, 2009.

38. Haigis MC and Guarente LP: Mammalian sirtuins--emerging roles in physiology, aging, and calorie restriction. Genes Dev 20 2913-2921, 2006.
39. Jeong SM, Xiao C, Finley LW, Lahusen T, Souza AL, Pierce K, Li YH, Wang X, Laurent G, German NJ, et al: SIRT4 has tumor-suppressive activity and regulates the cellular metabolic response to DNA damage by inhibiting mitochondrial glutamine metabolism. Cancer Cell 23: 450-463, 2013.

40. Shi Q, Liu T, Zhang X, Geng J, He X, Nu M and Pang D Decreased sirtuin 4 expression is associated with poor prognosis in patients with invasive breast cancer. Oncol Lett 12: 2606-2612, 2016.

41. Qi L and Ding Y: Involvement of the CREB5 regulatory network in colorectal cancer metastasis. Yi Chuan 36: 679-684, 2014.

42. Harada Y, Tamura Y and Endo T: Identification of yeast Art5 as a multicopy suppressor for the mitochondrial translocator maintenance protein Tam41. Biochem Biophys Res Commun 392: 228-233, 2010

43. Shih YL, Chou HM, Chou HC, Lu HF, Chu YL, Shang HS and Chung JG: Casticin impairs cell migration and invasion of mouse melanoma B16F10 cells via PI3K/AKT and NF- $\kappa \mathrm{B}$ signaling pathways. Environ Toxicol 32: 2097-2112, 2017.

44. Wang J, Wang L, Ho CT, Zhang K, Liu Q and Zhao H: Garcinol from Garcinia indica downregulates cancer stem-like cell biomarker ALDH1A1 in nonsmall cell lung cancer A549 cells through DDIT3 activation. J Agric Food Chem 65: 3675-3683, 2017.

45. Čokić VP, Smith RD, Biancotto A, Noguchi CT, Puri RK and Schechter AN: Globin gene expression in correlation with $\mathrm{G}$ protein-related genes during erythroid differentiation. BMC Genomics 14: 116, 2013

46. Lian Z, Han J, Huang L, Wei C, Fan Y, Xu J, Zhou M, Feng H, Liu Q, Chen L, et al: A005, a novel inhibitor of phosphatidylinositol 3-kinase/mammalian target of rapamycin, prevents osteosarcomainduced osteolysis. Carcinogenesis, 2018.

47. Yu G, Liu G, Yuan D, Dai J, Cui Y and Tang X: Long non-coding RNA ANRIL is associated with a poor prognosis of osteosarcoma and promotes tumorigenesis via PI3K/Akt pathway. J Bone Oncol 11: 51-55, 2018

48. Chen L, Pei H, Lu SJ, Liu ZJ, Yan L, Zhao XM, Hu B and Lu HG: SPOP suppresses osteosarcoma invasion via PI3K/AKT/NF- $\kappa \mathrm{B}$ signaling pathway. Eur Rev Med Pharmacol Sci 22: 609-615, 2018.

49. Hu B, Lv X, Gao F, Chen S, Wang S, Qing X, Liu J, Wang B and Shao Z: Downregulation of DEPTOR inhibits the proliferation, migration, and survival of osteosarcoma through PI3K/Akt/ mTOR pathway. OncoTargets Ther 10: 4379-4391, 2017.

50. Pu Y, Yi Q, Zhao F, Wang H, Cai W and Cai S: MiR-20a-5p represses multi-drug resistance in osteosarcoma by targeting the KIF26B gene. Cancer Cell Int 16: 64, 2016.

51. Fujimori A, Cheng SL, Avioli LV and Civitelli R: Structurefunction relationship of parathyroid hormone: Activation of phospholipase- $\mathrm{C}$, protein kinase-A and -C in osteosarcoma cells. Endocrinology 130: 29-36, 1992.

52. Miles RR, Sluka JP, Halladay DL, Santerre RF, Hale LV, Bloem L, Thirunavukkarasu K, Galvin RJ, Hock JM and Onyia JE: ADAMTS-1: A cellular disintegrin and metalloprotease with thrombospondin motifs is a target for parathyroid hormone in bone. Endocrinology 141: 4533-4542, 2000. 